\title{
Micromechanics of the Deformation and Damage of Steel Fiber-reinforced Concrete
}

\author{
A. OUAar,,${ }^{1, *}$ I. Doghri, ${ }^{1}$ L. Delannay ${ }^{1}$ AND J.-F. Thimus ${ }^{2}$ \\ ${ }^{1}$ Université Catholique de Louvain (UCL) CESAME \\ Bâtiment Euler, 4 Avenue G. Lemaître B-1348 \\ Louvain-la-Neuve, Belgium \\ ${ }^{2}$ Université Catholique de Louvain (UCL) Département AUCE \\ Bâtiment Vinci, Place Sainte-Barbe B-1348 \\ Louvain-la-Neuve, Belgium
}

\begin{abstract}
This article presents a study of steel fiber-reinforced concrete (SFRC). In its first part, a four-point bending test performed on both plain concrete and SFRC is investigated. The collected nonlinear load-deflection curves are transformed into stress-strain curves with the help of an incremental method, which the authors developed in the nonlinear regime. In the second part of this article, the authors present a micromechanical approach based on Mori-Tanaka/ Voigt mean-field homogenization schemes in order to model the effective nonlinear behavior of the three-phase brittle composite materials. The first phase (concrete matrix) is assumed to obey Ju's brittle damage model. The second phase (fibers) is modeled with classical $J_{2}$ plasticity, while the third phase represents cavities. Numerical algorithms enable the simulation of SFRC within reasonable CPU time and memory requirements. The homogenization module is interfaced to the finite element package ABAQUS. A two-scale simulation of the bending test is validated against the experimental results.
\end{abstract}

KEY WORDS: micromechanics, homogenization, damage, experiments, steel fiberreinforced concrete.

\section{INTRODUCTION}

$\mathrm{C}$ OMPOSITE MATERIALS REINFORCED with particles or fibers are widely used in industrial applications due to their good mechanical,

\footnotetext{
*Author to whom correspondence should be addressed. E-mail: ouaar@mema.ucl.ac.be Figures 7, 21 and 23 appear in color online: http://ijd.sagepub.com
}

International Journal of DAMAGE Mechanics, Vol. 16-April 2007 
thermal, and electrical properties. For the scientist as well as for industry, an important challenge is to understand the relationship between microstructure and macroscopic response. Numerical simulation is a necessary tool in order to design composite materials with optimized properties.

Concrete lends itself to a variety of innovative designs, but two characteristics have limited its use: it is brittle and weak in tension. One way to overcome this problem is to add metallic fibers to the concrete mix. Fibers hinder microcracking, thus improving the composite's ductility (Rossi, 1998). Steel fiber-reinforced concrete (SFRC) captivates one's interest because it has a large range of applications in civil engineering. It is used in bridges, pipes, airport runways, tunnel linings, etc. Steel fibers are much stiffer than concrete. However, as illustrated in the present study, the addition of fibers may induce porosity that reduces significantly the overall stiffness; see also Walkus et al. (1979).

The SFRC has been the subject of previous investigations. Among researchers who focused on experimental aspects, Walkus et al. (1979) analyzed the response of concrete reinforced with various volume fractions of fibers under tensile loading. Soroushian and Bayasi (1991) performed bending tests, and Van Hauwaert (2000) investigated the fiber adhesion. The mechanical response of concrete microstructures has also been studied numerically, but the use of homogenization methods has generally been limited to the elastic regime. For example, Li et al. (1999) and Zaho and Chen (1998) used an extended double inclusion scheme based on the Eshelby (1957) equivalent inclusion to model the interfacial transition zone (ITZ). The latter represents the interface between coarse aggregates and cement paste. Teng et al. (2004) predicted the composite's elastic response using a tensorial representation of fiber orientation.

In the present article, the authors develop a micromechanical approach based on mean-field homogenization in order to predict the nonlinear behavior of SFRC. Nonlinearity results from damage in the concrete matrix and from the interaction with elastic-plastic metallic fibers. The authors study some experimental, (micro)mechanical, and numerical aspects of SFRC with the aim of achieving an appropriate modeling of the composite.

The experimental study led to the crucial finding that initial voids and microcracks coalescence due to damage process, and porosity due to fiber addition must not be confused even if both cause a decrease in the composite's stiffness. Assuming that concrete material, including microcracks and initial voids, represents one phase (the matrix) and fibers the other (reinforcement phase), when these two phases are mixed in order to obtain SFRC, the initial porosity of the matrix phase increases in a significant manner. This porosity increase is viewed as a full phase and the SFRC is modeled, therefore, as a three-phase composite. 
The first phase consists of concrete material, microcracks and initial voids; the second phase represents fibers, while the third one represents the increase in porosity.

Activation of microcracks and coalescence of voids is handled by Ju's (1989) energy-based brittle damage model, which accounts for damage evolution only when the material is submitted to tensile strains. The latter are extracted using spectral decomposition and positive projection tensors (Krajcinovic, 1996).

The SFRC is modeled with an incremental formulation of Mori-Tanaka/ Voigt mean-field homogenization schemes in the nonlinear regime; see Doghri and Ouaar (2003), Doghri and Friebel (2005) and Doghri and Tinel (2005). In order to simulate the four-point bending test and other more elaborated structures of civil engineering, a two-scale approach was developed based on an interface between the authors homogenization module and the ABAQUS (2004) finite element (FE) software through its user-defined material capability.

The article is organized as follows. The 'Experimental bending tests' section deals with experimental four-point bending tests on both plain concrete and SFRC. Composite samples are prepared with two values of the fiber volume fraction. To be workable, experimental load-deflection curves are converted into stress-strain curves in 'Conversion of the loaddeflection curve into a stress-strain curve' section. Conversion is based on the assumption of pure bending and on an incremental formulation, which relates bending moment and normal stress acting over the cross section of the beam. The 'Homogenization procedure' section presents the two-step homogenization approach based on Mori-Tanaka and Voigt schemes. The constitutive models used at the microlevel (Ju's brittle damage model for the concrete matrix and $J_{2}$ elastoplasticity for the steel fibers) are presented in the 'Micro constitutive models' section. The overall micromacro algorithm is outlined in the 'Overall micro-macro algorithm' section, while the 'Numerical simulation' section is devoted to numerical results. Model predictions are compared to experimental results, demonstrating the necessity to give specific treatment to the initial porosity and to the voids created by damage.

\section{EXPERIMENTAL BENDING TESTS}

Mechanical properties of concrete and SFRC have been assessed using a four-point bending apparatus. In order to evaluate the reinforcement by steel fibers, the same tests have been conducted on three series of specimen: (i) plain concrete, (ii) SFRC containing $1 \%$ volume of fibers, and (iii) SFRC containing 3\% volume of fibers. 
Table 1. Ingredient proportions of the concrete mixture.

\begin{tabular}{lc}
\hline Constituent & Quantity (kg) \\
\hline Cement 32.5 & 18.5 \\
River sand 0/5 & 37.2 \\
Coarse aggregates $4 / 14$ & 55.8 \\
Water & 8.37 \\
\hline
\end{tabular}

All specimens have been cast in $600 \times 150 \times 150 \mathrm{~mm}$ steel molds, in accordance with the recommendations of JSCE-SF4, NBN B15-238, and ASTM C1018. Bending tests were performed after 28 days, when concrete was assumed to have reached its maximum strength. The type of fibers used in this investigation is hooked-end steel. The fibers, called ZP30/0.5 are manufactured by Bekaert (Belgium). Fibers have a length of $30 \mathrm{~mm}$ with a diameter of $0.5 \mathrm{~mm}$. Hence, the aspect ratio $A_{r}$ is 60 . Mechanical properties are the following: Young's modulus $E=210 \mathrm{GPa}$, and Poisson's ratio $v=0.3$. The concrete mix was prepared with special cement 32.5 (LAFARGE MORTIERS), river sand $0 / 5$, coarse aggregates $4 / 14$, and water. Table 1 summarizes the ingredient proportions of the mixture.

Bending tests have been conducted on an MTS testing machine equipped with a load cell of $100 \mathrm{kN}$. The load is applied to the specimen through two rollers, $150 \mathrm{~mm}$ apart (Figure 1). Two linear voltage differential transformers (LVDT) are fixed on the lateral faces of the specimen. They record the vertical deflection of the beam. A MTS 632.02F-20 extensometer (or 'clip on gage') is pasted underneath the beam in order to measure the stretching of lower fibers. Near the extensometer, a stress gage of type CEA-06-250UN-350 provides supplementary strain measurement of the lower fibers. Figure 1 shows a schematic representation of the various components of the acquisition system.

Load-deflection curves for both plain concrete and concrete reinforced with 1 or $3 \%$ of steel fibers are presented in Figure 2. Three specimens are tested for each case. It appears that fibers increase the ductility of concrete: the area located under load-deflection curves is significantly raised. On the other hand, the presence of fibers decreases somewhat the overall stiffness. Adding fibers increases the porosity, which counterbalances the reinforcement of the composite. This conclusion has a crucial importance in the micromechanical modeling of the composite. Measurements of the maximum tensile strain underneath the beam are not shown here. They are less accurate, and will therefore only be used in a qualitative comparison with the deflection measurements (next section). 


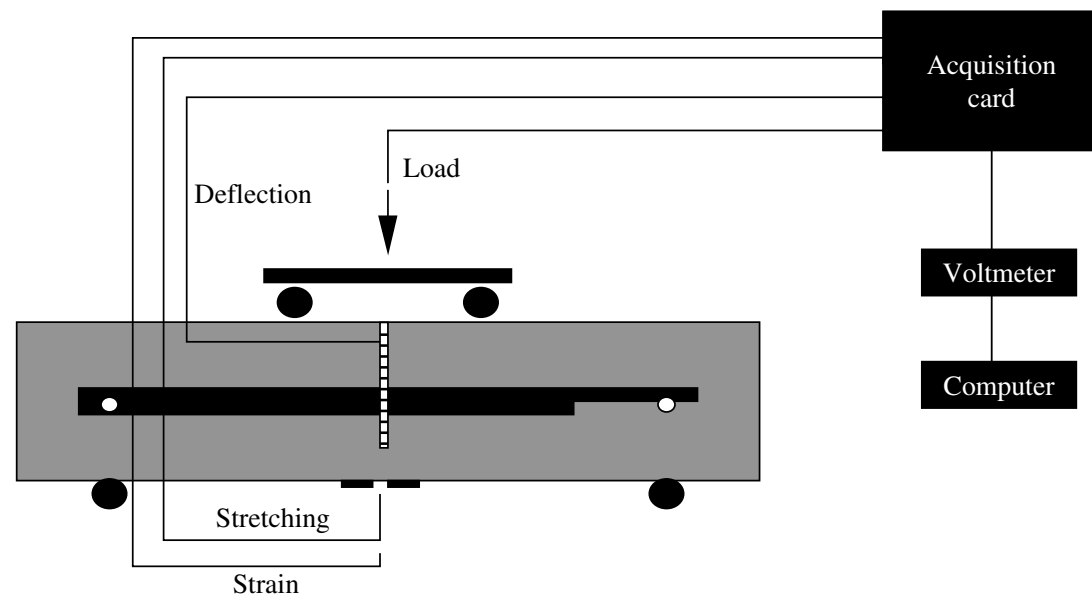

(a)

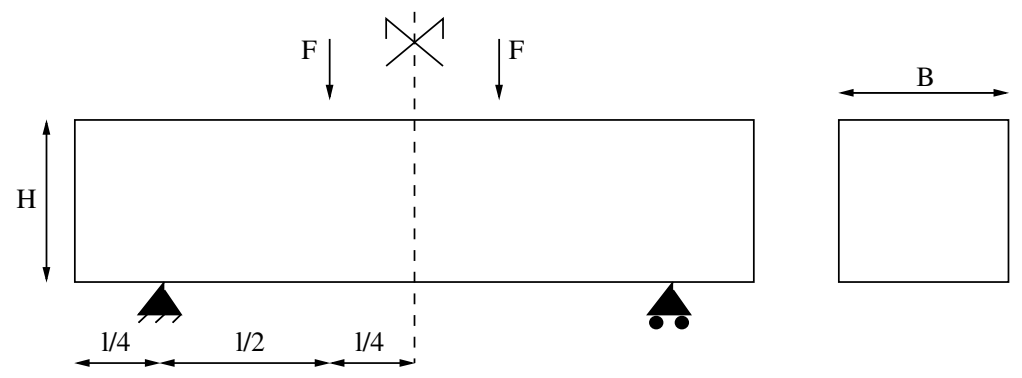

(b)

Figure 1. Four-point bending test: (a) schematic representation of the acquisition chain and (b) beam model and notations.

\section{CONVERSION OF THE LOAD-DEFLECTION CURVE INTO A STRESS-STRAIN CURVE}

Due to its poor tensile strength, concrete is generally characterized by bending tests instead of uniaxial tensile tests. Uniaxial stress-strain curves are, nevertheless, necessary in order to fit the parameters of constitutive models in numerical simulations. In this section, the load-deflection curve is exploited under simplifying assumptions. These assumptions will be validated in a subsequent section using FE modeling. According to classical beam theory, four-point bending creates a constant bending moment $M$ throughout the portion of the beam located between the two central rollers. 


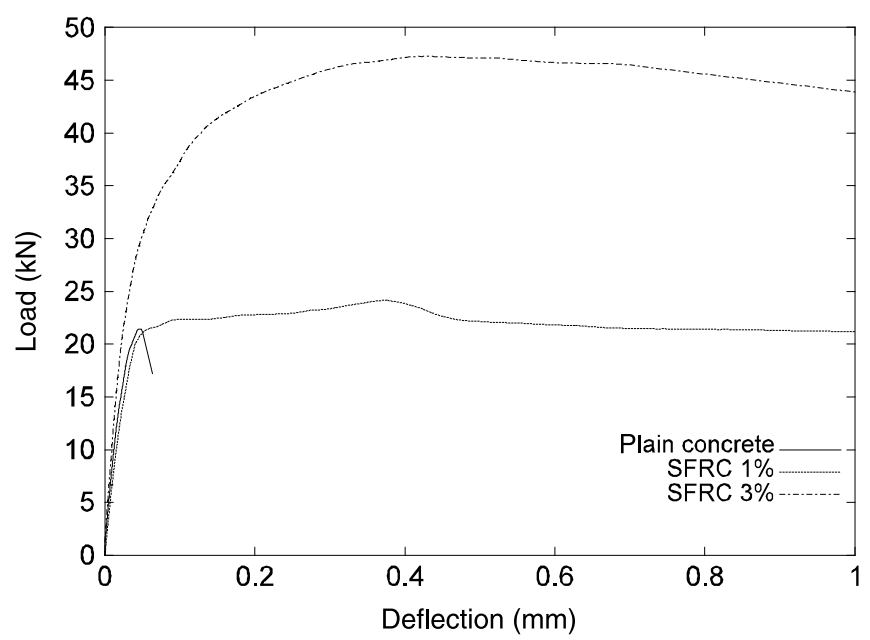

Figure 2. Plain concrete and SFRC (1 and 3\%) under four-point bending: load-deflection curve.

For the apparatus sketched in Figure 1, one obtains $M=F l / 2$, where $F$ is the load $F$ exerted on one roller. The authors consider, for the moment, that all components of the stress tensor are negligible compared to the longitudinal stress $\sigma_{x x}$, and also neglect friction. Hence, bending occurs under the action of a pure bending moment. If $y \in[-C, H-C]$ represents the heightcoordinate of the cross section and $B$, the thickness in the $z$ direction:

$$
M=B \int_{-C}^{H-C} \sigma_{x x}(y) y \mathrm{~d} y=F \frac{l}{2} .
$$

As friction is neglected, the resultant of $\sigma_{x x}$ over the short-transverse section of the beam is nil:

$$
B \int_{-C}^{H-C} \sigma_{x x}(y) \mathrm{d} y=0 .
$$

Pure bending deforms the longitudinal material lines into concentric arcs. In Figure 3, the neutral line $y=0$ separates two regions of the beam, $y \in[-C, 0]$ and $y \in[0, H-C]$ inside which $\sigma_{x x}$ is compressive and tensile, respectively. The longitudinal strain $\varepsilon_{x x}$ varies linearly with $y$. If $R$ denotes the curvature radius of the neutral line, and if $C$ represents the distance of the neutral line relative to the upper fiber of the beam, one writes:

$$
\varepsilon_{x x}(y)=\frac{y}{R}=\frac{y}{H-C} \varepsilon_{t} .
$$




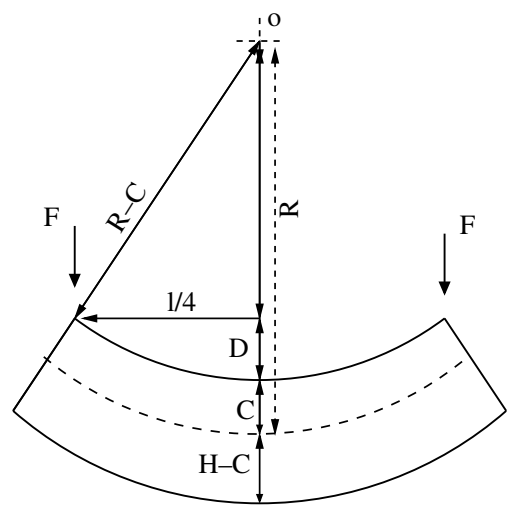

Figure 3. Radius of curvature-deflection relationship.

In the right-hand side expression, $\varepsilon_{t}$ is the maximum tensile strain. It can be measured by the gage placed underneath the beam. However, as mentioned in the previous section, the data recorded by that gage are not accurate enough. It is better to measure the beam's vertical deflection $D$, compute the bending radius $R$ from the following relation (see Figure 3):

$$
R=\frac{1}{2 D}\left[\left(\frac{l}{4}\right)^{2}+D^{2}\right]+C,
$$

and obtain $\varepsilon_{t}=(H-C) / R$. Designating successive bending increments by the subscript $i$, it is shown hereafter how using the measured values of $M_{i}$ and $D_{i}$ together with the pure bending assumption, one is able to derive the longitudinal stress-strain relationship in an incremental manner.

During the first loading steps, $D_{i}$ evolves linearly with $M_{i}$ because the material remains elastic. By substituting Equation (3) into (2) and using the linear elastic relation, $\sigma_{x x}=k_{i} \varepsilon_{x x}$, it turns out that the neutral line coincides with the mid-thickness (Figure 4), i.e., $C_{i}=H / 2$. Based on Equations (1)-(3), the bending moment $M_{i}$ is related to the bending radius $R_{i}$ and to the yet unknown elastic constant $k_{i}$ :

$$
\frac{M_{i}}{B}=\int_{-H / 2}^{H / 2} k_{i} \frac{y}{R_{i}} y \mathrm{~d} y=\frac{k_{i} H^{3}}{12 R_{i}}=\frac{k_{i} H^{2}}{6} \varepsilon_{i t} .
$$

In fact, the treatment of elastic bending does not require several increments because the value of $k_{i}$ is constant. Let us thus consider a single bending increment in the elastic regime: $M_{1}$ now refers to the 


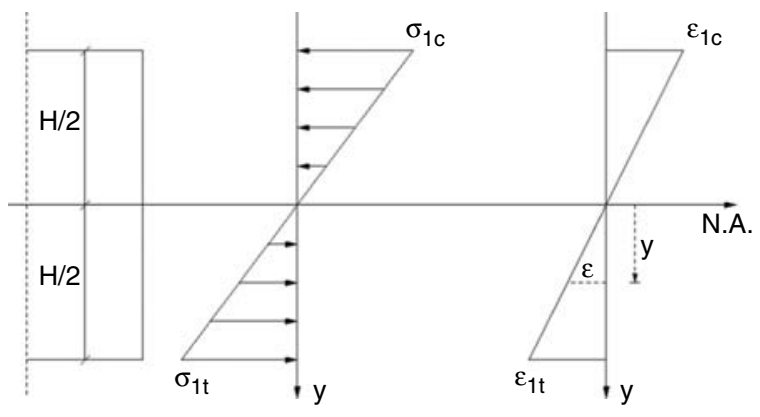

(a)

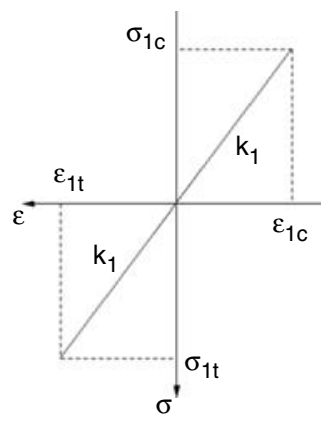

(b)

Figure 4. Stress and strain states over a cross section of a bent beam and stress-strain curve in the linear regime.

maximum bending moment for which $\varepsilon_{i t}$ and $D_{i}$ evolve linearly. When the bending moment is raised above $M_{1}$, the damage threshold is reached and the composite becomes less stiff in tension than compression. Fulfillment of Equation (2) requires that tensile stresses counterbalance compressive stresses, which imposes upwards displacement of the neutral line (Figure 5). The distribution of longitudinal stresses across the beam corresponds to:

$$
\begin{aligned}
& \sigma_{x x}=k_{1} \varepsilon_{x x} \quad \text { if } \quad y \in\left[-C_{2}, H_{1}\right], \\
& \sigma_{x x}=k_{1} \varepsilon_{1 t}+k_{2}\left(\varepsilon_{x x}-\varepsilon_{1 t}\right) \quad \text { if } \quad y \in\left[H_{1}, H-C_{2}\right],
\end{aligned}
$$

where $H_{1}$ is the value of $y$ for which $\varepsilon_{x x}=\varepsilon_{1 t}$ :

$$
H_{1}=R_{2} \varepsilon_{1 t}
$$

After this first nonlinear step, Equation (2) becomes:

$$
\int_{-C_{2}}^{H_{1}} k_{1} \frac{y}{R_{2}} \mathrm{~d} y+\int_{H_{1}}^{H-C_{2}}\left[\left(k_{1}-k_{2}\right) \varepsilon_{1 t}+k_{2} \frac{y}{R_{2}}\right] \mathrm{d} y=0,
$$

and the bending moment equation i.e., Equation (3) becomes:

$$
\frac{M_{2}}{B}=\int_{-C_{2}}^{H_{1}} k_{1} \frac{y}{R_{2}} y \mathrm{~d} y+\int_{H_{1}}^{H-C_{2}}\left[\left(k_{1}-k_{2}\right) \varepsilon_{1 t}+k_{2} \frac{y}{R_{2}}\right] y \mathrm{~d} y .
$$

Equations (8) and (9) form a set of coupled equations with two unknowns: $k_{2}$ and $C_{2}$. This equation set is solved iteratively, before 


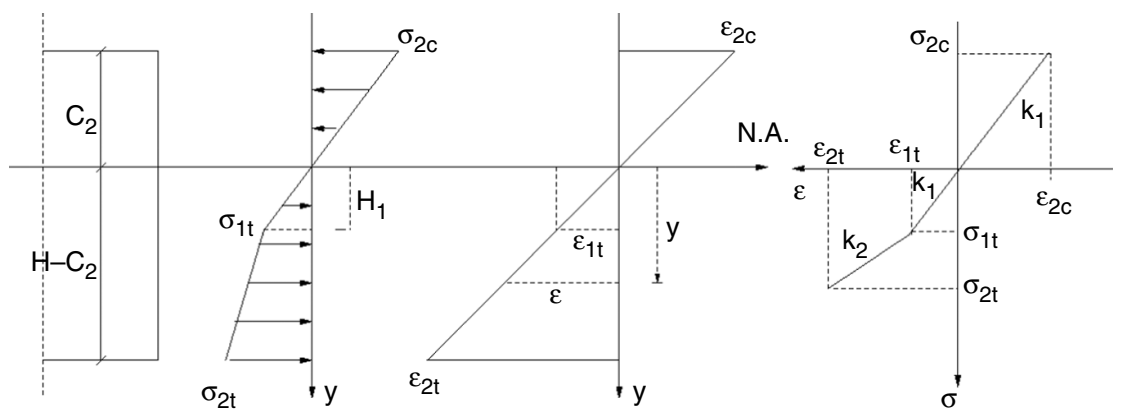

(a)

(b)

Figure 5. Stress and strain states over a cross section of a bent beam and stress-strain curve in the nonlinear regime.

moving on to the next bending increment. After the second nonlinear increment, the expression of the longitudinal stress is:

$$
\begin{aligned}
& \sigma_{x x}=k_{1} \varepsilon_{x x} \quad \text { if } \quad y \in\left[-C_{3}, H_{1}\right], \\
& \sigma_{x x}=k_{1} \varepsilon_{1 t}+k_{2}\left(\varepsilon_{x x}-\varepsilon_{1 t}\right) \quad \text { if } \quad y \in\left[H_{1}, H_{2}\right], \\
& \sigma_{x x}=k_{1} \varepsilon_{1 t}+k_{2}\left(\varepsilon_{2 t}-\varepsilon_{1 t}\right)+k_{3}\left(\varepsilon_{x x}-\varepsilon_{2 t}\right) \quad \text { if } \quad y \in\left[H_{2}, H-C_{3}\right],
\end{aligned}
$$

where $H_{1}=R_{3} \varepsilon_{1 t}$ and $H_{2}=R_{3} \varepsilon_{2 t}$. The unknowns $k_{3}$ and $C_{3}$ are determined by constructing an equation set similar to Equations (8) and (9) The same treatment is then applied to the following bending increments, leading to the uniaxial stress-strain curves shown in Figure 6. It is worth noting that these curves have been constructed by relying solely on two types of measurements: $D_{i}$ and $M_{i}$. Maximum tensile strains $\varepsilon_{i t}$ have been derived from $D_{i}$ and $C_{i}$ using Equations (3) and (4).

\section{HOMOGENIZATION PROCEDURE}

In this section, the authors present the key ideas behind the extension of some homogenization models to the rate-independent inelastic regime. For more details, see Doghri and Ouaar (2003).

\section{Rate-independent Models}

In the so-called incremental formulation proposed by Hill (1965), homogenization schemes are extended from linear elasticity to 


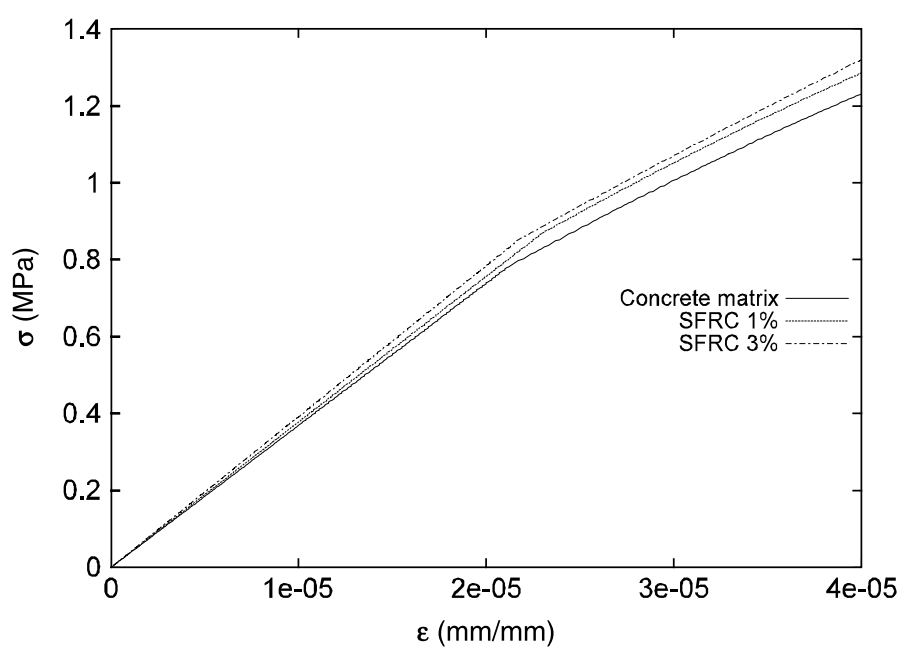

Figure 6. Tensile stress-strain curves of bent plain concrete and SFRC samples.

rate-independent inelastic behavior by relating stress and strain rates through an instantaneous tangent operator $\mathbf{C}^{t g}$, i.e.,

$$
\dot{\sigma}(\mathbf{x}, t)=\mathbf{C}^{t g}(\varepsilon(\mathbf{x}, t), t): \dot{\varepsilon}(\mathbf{x}, t) .
$$

Although form-similar to linear elasticity, the extension presents a major difference with the former because in the nonlinear regime, even if a phase is made of a homogeneous material its tangent operator is not uniform. A workaround is to define for each phase (domain $\omega_{r}$ ) a so-called reference material such that its operator $\hat{\mathbf{C}}_{r}^{t g}(t)$ is uniform in space and varies only with time. Consequently:

$$
\dot{\sigma}(\mathbf{x}, t)=\hat{\mathbf{C}}_{r}^{t g}(t): \dot{\varepsilon}(\mathbf{x}, t) \quad \forall x \in \omega_{r} .
$$

In this work, the reference moduli $\hat{\mathbf{C}}_{r}^{t g}(t)$ in a phase $r$ are computed by calling the constitutive box of the phase's real material with the phaseaveraged strains and strain rates as arguments.

\section{Two-phase Composites}

Consider a representative volume element (RVE) of a two-phase composite (domain $\omega$ ) made of a matrix phase $\left(\omega_{0}\right)$ reinforced with aligned inclusions $\left(\omega_{1}\right)$ having similar shape, orientation, and material. 
The RVE-averaged macro stress rate is obtained from the phase-averaged stress rates as follows:

$$
<\dot{\sigma}>_{\omega}=v_{0}<\dot{\sigma}>_{\omega_{0}}+v_{1}<\dot{\sigma}>_{\omega_{1}},
$$

where $v_{0}$ and $v_{1}=1-v_{0}$ are the volume fractions of the matrix and inclusion phases, respectively. The macro stress and strain rates can also be related through the overall tangent operator $\overline{\mathbf{C}}^{\text {tg }}$ :

$$
<\dot{\sigma}>_{\omega}=\overline{\mathbf{C}}^{t g}:<\dot{\varepsilon}>_{\omega} .
$$

A given homogenization model can be defined by the corresponding expression of a so-called strain concentration tensor $\mathbf{A}$, which relates the average strain rate in the inclusion phase to the RVE-averaged strain rate:

$$
<\dot{\varepsilon}>_{\omega_{1}}=\mathbf{A}:<\dot{\varepsilon}>_{\omega} .
$$

The following expression of the overall tangent operator is then obtained:

$$
\overline{\mathbf{C}}^{t g}=\hat{\mathbf{C}}_{0}^{t g}+v_{1}\left(\hat{\mathbf{C}}_{1}^{t g}-\hat{\mathbf{C}}_{0}^{t g}\right): \mathbf{A} .
$$

An efficient model is the one proposed by Mori and Tanaka (1973). It is based on the well-known Eshelby (1957) equivalent inclusion problem and can be interpreted as follows (Benveniste, 1987): each inclusion in the composite behaves like an isolated inclusion in the matrix seeing the average strain in the matrix as a far-field strain. The expression of $\mathbf{A}$ for the Mori-Tanaka model is the following:

$$
\mathbf{A}=\left[\left(1-v_{1}\right)\left\{\mathbf{I}^{s}+\mathbf{S}_{\left(I, \hat{\mathbf{C}}_{0}^{t g}\right)}:\left[\left(\hat{\mathbf{C}}_{0}^{t g}\right)^{-1}: \hat{\mathbf{C}}_{1}^{t g}-\mathbf{I}^{s}\right]\right\}+v_{1} \mathbf{I}^{s}\right]^{-1},
$$

where $\mathbf{I}^{s}$ is the fourth-rank symmetric identity tensor and $\mathbf{S}$ Eshelby's tensor. The latter depends on the matrix reference tangent moduli $\hat{\mathbf{C}}_{0}^{t g}$ and the shape and orientation of inclusions (I). For isotropic moduli and a spheroid (an ellipsoid with a revolution axis), $\mathbf{S}$ depends only on the instantaneous Poisson's ratio and the aspect ratio $A_{r}$.

In the case of cavities instead of solid inclusions, $\mathbf{A}$ is obtained from the limiting case $\hat{\mathbf{C}}_{1}^{t g} \rightarrow 0$ :

$$
\mathbf{A}=\left[\mathbf{I}^{S}-v_{0} \mathbf{S}_{\left(I, \hat{\mathbf{C}}_{0}^{t g}\right)}\right]^{-1} .
$$




\section{Multiphase Composites}

As suggested by the experimental study, SFRC must be modeled as a three-phase composite. The matrix phase is concrete including microcracks and initial voids. Steel fibers constitute a second phase, while cavities due to increased porosity represent the third phase. The method described hereafter can be applied to a general multiphase composite (domain $\Omega$ ) made of a matrix material $\left(\Omega_{0}\right)$ and $N$ families of spheroidal inclusions or cavities. Each family $(i)$ is characterized by an aspect ratio, an orientation distribution function (ODF) $\Psi_{i}(p)$, and a material for solid inclusions. The orientation of an individual spheroid is determined by a unit vector $p$ along the revolution axis. The volume fractions of the matrix phase and the families of inclusions are designated by $v_{0}$ and $v_{i}$, respectively, $v_{0}+\sum_{i=1}^{N} v_{i}=1$.

In this article, the multiphase RVE is homogenized using the aggregate decomposition approach advocated by Camacho et al. (1990), Lielens (1999), and Pierard et al. (2004) in linear thermoelasticity, and Doghri and Tinel (2005) in elastoplasticity. The method starts with a virtual decomposition step by defining so-called pseudo-grains $\Omega_{i}, p$, each containing matrix material (in concentration $v_{0}$ ) and all inclusions of family ( $i$ ) whose orientation is between $p$ and $p+\mathrm{d} p$ (Figure 7 step 1). Next (Figure 7 step 2) each pseudo-grain is homogenized using a

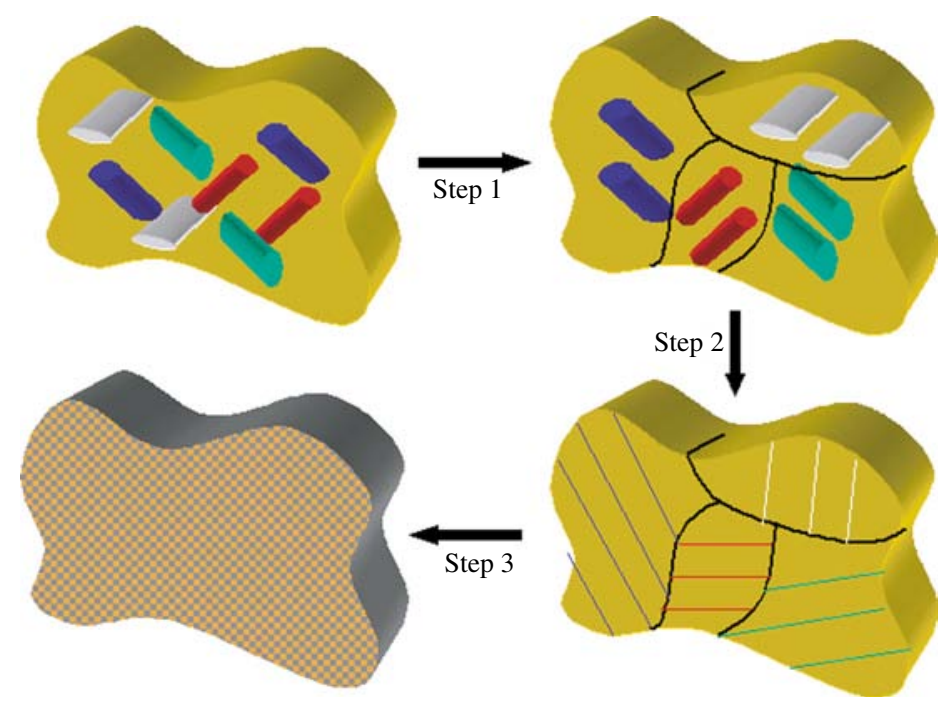

Figure 7. Aggregate decomposition approach for multiphase composites. 
model suitable for two-phase composites, typically Mori-Tanaka (the previous section). Finally, the set of homogenized pseudo-grains is itself homogenized (Figure 7 step 3) using a simple Voigt model in this work.

It can be shown that the volume average of the stress rate over the RVE can be expressed as follows:

$$
\langle\dot{\sigma}\rangle_{\Omega}=\sum_{i=1}^{N} \frac{v_{i}}{1-v_{0}} \oint\langle\dot{\sigma}\rangle_{\Omega i, p} \psi_{i}(p) \mathrm{d} p=\left\langle\langle\dot{\sigma}\rangle_{\Omega i, p}\right\rangle_{i, \psi_{i}} .
$$

This can be rewritten as follows using the homogenized tangent operators of the RVE and of each pseudo-grain:

$$
\overline{\mathbf{C}}^{t g}:<\dot{\varepsilon}>_{\Omega}=\left\langle\overline{\mathbf{C}}_{\Omega i, \mathbf{p}}^{t g}:<\dot{\varepsilon}>_{\Omega_{i}, \mathbf{p}}\right\rangle_{i, \psi_{i}} .
$$

Using the Voigt model in the final step supposes that the average strain rates in all pseudo-grains are the same, which gives the RVE's overall tangent operator as follows:

$$
\overline{\mathbf{C}}^{t g}=\left\langle\overline{\mathbf{C}}_{\Omega i, \mathbf{p}}^{t g}\right\rangle_{i, \psi_{i}} .
$$

\section{Eshelby's Tensor Computation}

For nonlinear models, tangent operators are anisotropic, therefore Eshelby's tensor ('Two-phase composites' section) has to be computed numerically (Gavazzi and Lagoudas, 1990). However, it can be shown that the resulting predictions of the effective response are too stiff. A workaround is to compute Eshelby's tensor with an isotropic part of the matrix' tangent operator. For an anisotropic operator $\mathbf{C}^{\text {ani }}$, its isotropic part $\mathbf{C}^{\text {iso }}$ is defined by the tangent bulk and shear moduli $\kappa_{t}$ and $\mu_{t}$. A general method used by Bornert et al. (2001) and Doghri and Ouaar (2003) gives the following expressions:

$$
\kappa_{t}=\frac{1}{9} \mathrm{C}_{\mathrm{lljj}}^{\mathrm{ani}}, \quad \mu_{t}=\frac{1}{10}\left(\mathrm{C}_{\mathrm{illi}}^{\mathrm{ani}}-3 \kappa_{t}\right) .
$$

Actually, for a spheroid, Eshelby's tensor depends on the tangent Poisson's ratio:

$$
v_{t}=\frac{3 \kappa_{t}-2 \mu_{t}}{6 \kappa_{t}+2 \mu_{t}} .
$$




\section{MICRO CONSTITUTIVE MODELS}

In this work, two mechanical models are considered at the microlevel. The authors assume that the matrix material (concrete) obeys the anisotropic brittle damage model of Ju (1989) while the steel inclusions follow a classical $J_{2}$ elastoplastic model.

\section{$J_{2}$ Elastoplasticity}

Hereafter the expressions of the tangent operators for $J_{2}$ elastoplasticity are given (see chapter 12 in Doghri (2000) for details). Two operators are defined: an elastoplastic or 'continuum' $\mathbf{C}^{\mathrm{ep}}$ and an algorithmic or 'consistent' $\mathbf{C}^{\text {alg }}$. The former relates infinitesimal stress and strain rates, while the latter is obtained from consistent linearization of the timediscretization algorithm. The following expressions are found:

$$
\mathbf{C}^{\mathrm{ep}}=\mathbf{C}^{\mathrm{el}}-\frac{(2 \mu)^{2}}{h} N \otimes N, \quad \mathbf{C}^{\mathrm{alg}}=\mathbf{C}^{\mathrm{ep}}-(2 \mu)^{2}(\Delta p) \frac{\sigma_{\mathrm{eq}}}{\sigma_{\mathrm{eq}}^{\mathrm{tr}}} \frac{\partial N}{\partial \sigma},
$$

where $\mathbf{C}^{\mathrm{el}}$ is the isotropic Hooke's stiffness defined by elastic bulk and shear moduli $\kappa$ and $\mu$, and the following notation is used:

$$
h=3 \mu+\frac{\mathrm{d} R}{\mathrm{~d} p}, N=\frac{\partial f}{\partial \sigma}, f=\sigma_{\mathrm{eq}}-R(p)-\sigma_{Y} \leq 0,
$$

with $\sigma_{Y}$ being the initial yield stress, $f$ the yield function, $R(p)$ the isotropic hardening stress, $p$ the accumulated plastic strain, $\sigma_{\text {eq }}$ the von Mises stress, $\Delta p$ the increment of $p$, and $\sigma_{\text {eq }}^{\text {tr }}$ an elastic trial value of $\sigma_{\text {eq }}$. The tangent bulk and shear moduli defining the isotropic part of $\mathbf{C}^{\text {alg }}$ ('Eshelby's tensor compulation' section) are given by Doghri and Ouaar (2003):

$$
\kappa_{t}=\kappa, \quad \mu_{t}=\mu-\frac{3}{5} \mu^{2}\left[\frac{1}{h}+4 \frac{\Delta p}{\sigma_{\mathrm{eq}}^{\mathrm{tr}}}\right] .
$$

\section{Ju's Unilateral Damage Model}

In this article, the behavior of the brittle matrix material is modeled using the unilateral damage model of Ju (1989). In one-dimensional (1D) model, it can be understood by considering uniaxial tensile loading and unloading as displayed in Figure 8. The path $\mathbf{O A}$ is a straight line of slope $E$, Young's modulus of the material. Along this line, the material 


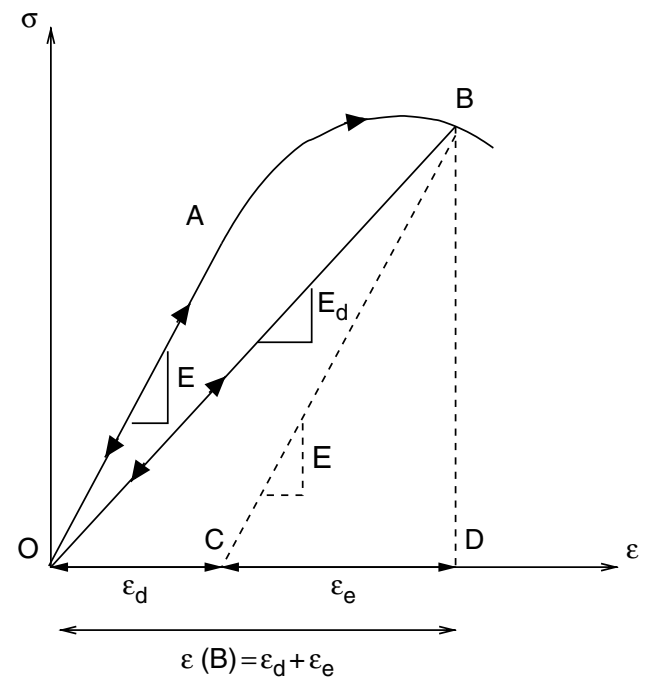

Figure 8. Uniaxial stress-strain curve of a damaged material.

exhibits an elastic behavior. Along the path $\mathbf{A B}$, the response becomes nonlinear, stress and strain are not proportional and damage takes place. If one unloads anywhere along $\mathbf{A B}$, the response will be along the straight line BO with a slope $E_{d}$ called damaged elastic modulus. If one reloads, the response will be along $\mathbf{O B}$ and damage will evolve again only when point $\mathbf{B}$ is reached. For multiaxial stress states, the evolution of the pattern of microcracks in brittle materials such as concrete or rocks is governed primarily by the principal strains or stresses. When such materials undergo tensile fields, microcracks are nucleated and propagated in planes which are orthogonal to the principal tensile stresses (Krajcinovic, 1996). In Ju's model, damage evolution is governed by principal tensile strains. First, one starts by computing the eigenvalues $\varepsilon_{i}$ and eigenvectors $e_{i}$ (forming an orthonormal and direct basis) of the total strain tensor $\varepsilon$. Therefore, the following spectral decomposition holds:

$$
\varepsilon=\sum_{i=1}^{3} \varepsilon_{i} e_{i} \otimes e_{i}, \quad e_{i} \cdot e_{j}=\delta_{i j},
$$

where $\delta_{i j}$ designates Kronecker's symbol. Next, the following positive projection operators are defined:

$$
\mathbf{Q}^{+}(\varepsilon)=\sum_{i=1}^{3} \hat{H}\left(\varepsilon_{i}\right) e_{i} \otimes e_{i}, \quad P_{i j k l}^{+}\left(\mathbf{Q}^{+}\right)=\frac{1}{2}\left(Q_{i k}^{+} Q_{j l}^{+}+Q_{i l}^{+} Q_{j k}^{+}\right),
$$


where $\mathbf{Q}^{+}$and $\mathbf{P}^{+}$are symmetric second- and fourth-rank tensors, respectively, and $\hat{H}\left(\varepsilon_{i}\right)$ represents the Heaviside ramp function: $\hat{H}\left(\varepsilon_{i}\right)=1$ if $\varepsilon_{i}>0$ and $\hat{H}\left(\varepsilon_{i}\right)=0$ otherwise. The positive or tensile part of the strain tensor can now be defined as follows:

$$
\varepsilon^{+}(\varepsilon)=\mathbf{P}^{+}: \varepsilon .
$$

Note that this relation is nonlinear, because $\mathbf{P}^{+}$depends on $\varepsilon$. It is easily checked that if all three principal strains $\varepsilon_{i}$ are tensile, then $Q_{i j}^{+}=\delta_{i j}$, $\mathbf{P}^{+}=\mathbf{I}^{s}$, and $\varepsilon^{+}=\varepsilon$. However, if all $\varepsilon_{i}$ are compressive then $\varepsilon^{+}=0$ and no damage will be predicted in the model described hereafter. For brittle damage, Ju defines a characteristic damage measure $\xi\left(\varepsilon^{+}\right)$as follows:

$$
\xi\left(\varepsilon^{+}\right) \equiv \frac{1}{2} \varepsilon^{+}: \mathbf{C}^{\mathrm{el}}: \varepsilon^{+},
$$

where $\mathbf{C}^{\mathrm{el}}$ is the initial (undamaged) elastic stiffness. The damage state is governed by the following criterion:

$$
g \equiv \tilde{G}(\xi)-r \leq 0
$$

The criterion can be viewed as a competition between available and suitable energies for damage evolution. As long as $\tilde{G}(\xi)$ is smaller than the damage threshold $r$, damage does not evolve. When damage evolves, it is seen that:

$$
\dot{r}=\dot{\xi} H(\xi), \quad H(\xi) \equiv \frac{\mathrm{d} \tilde{G}(\xi)}{\mathrm{d} \xi}
$$

In this article, the following expression for the damage evolution function $H(\xi)$ is used:

$$
H(\xi)=\left(k+\frac{1}{2}\right) A B \exp \left[B\left(\xi_{0}-\xi\right)\right]
$$

where $k$ represents the microcrack density, $\xi_{0}$ the initial characteristic damage measure; $A$ and $B$ are material-dependent parameters. The stressstrain relation of a damaged material in a secant or total deformation formulation is:

$$
\sigma=\mathbf{C}^{d}: \varepsilon
$$


where $\mathbf{C}^{d}$ is the secant anisotropic damaged operator. Its evolution equation is proposed by $\mathrm{Ju}$ as follows:

$$
\dot{\mathbf{C}}^{d}=-\dot{\xi} H(\xi) \mathbf{P}^{+}: \mathbf{C}^{\mathrm{el}}: \mathbf{P}^{+} .
$$

This equation indicates that only principal tensile strains acting in their associated directions contribute to damage evolution by microcrack growth. However, as pointed out by Chaboche (1992), under complex multiaxial loadings, Ju's model may exhibit a stress discontinuity in the stress-strain response, due to loss of symmetry of the damaged operator.

From a numerical point of view, consider a time interval $\left[t_{n}, t_{n+1}\right]$. The data are history variables at $t_{n}$ and the strain $\varepsilon_{n+1}$ at time $t_{n+1}$. Ju's model was implemented with the algorithm described hereafter.

- Spectral decomposition of $\varepsilon_{n+1}$ Equation (27).

- Second-rank positive projector $\mathbf{Q}^{+}\left(\varepsilon_{n+1}\right)$ Equation (28a).

- Fourth-rank positive projector $\mathbf{P}^{+}\left(\mathbf{Q}_{n+1}^{+}\right)$Equation (28b).

- Tensile strain tensor $\varepsilon^{+}\left(\varepsilon_{n+1}\right)$ Equation (29).

- Characteristic damage measure $\xi\left(\varepsilon_{n+1}^{+}\right)$Equation (30).

- Damage evolution function $H\left(\xi_{n+1}\right)$ Equation (33).

- Damage function $\tilde{G}\left(\xi_{n+1}\right)$ Equation (32).

- If $\tilde{G}\left(\xi_{n+1}\right)-r_{n} \leq 0$, damage did not evolve: $\mathbf{C}_{n+1}^{d}=\mathbf{C}_{n}^{d}$,

- Else, damage evolution: $r_{n+1}=\tilde{G}\left(\xi_{n+1}\right)$ and $\mathbf{C}_{n+1}^{d}$ is obtained by an implicit backward-Euler time-discretization of Equation (35), i.e.,

$$
\mathbf{C}_{n+1}^{d}=\mathbf{C}_{n}^{d}-\left(\xi_{n+1}-\xi_{n}\right) H_{n+1} P_{n+1}^{+}: \mathbf{C}^{\mathrm{el}}: P_{n+1}^{+}
$$

- Stress update: $\sigma_{n+1}=\mathbf{C}_{n+1}^{d}: \varepsilon_{n+1}$.

Ju's model defines a secant operator $\mathbf{C}^{d}$ while the incremental formulation of homogenization schemes needs tangent moduli $\mathbf{C}^{\text {dtg }}$ ('Homogenization procedure' section). The latter were approximated using a perturbation method.

\section{OVERALL MICRO-MACRO ALGORITHM}

In this section, the authors show how the models presented in 'Homogenization procedure' and 'Micro consistutive models' sections are combined together in an algorithmic procedure in order to homogenize a three-phase composite, where the matrix obeys Ju's brittle damage model, the steel fibers follow $J_{2}$ elastoplasticity, and there is also a cavity phase. 
For a time interval $\left[t_{n}, t_{n+1}\right]$, what one needs to compute are the macro stress $\bar{\sigma}$ and the overall tangent stiffness $\overline{\mathbf{C}}^{t g}$. The data are $\bar{\varepsilon}_{n}$ (macro strain at $t_{n}$ ), $\Delta \bar{\varepsilon}$ (macro strain increment), and history variables for each solid phase at $t_{n}$. In the following, in order to simplify the notation, the domain of a pseudo-grain $\Omega_{i, p}$ is designated by $\omega$ and its constituents are denoted by $\omega_{0}$ for the matrix and $\omega_{1}$ for the inclusions.

- Loops overall pseudo-grains.

- Voigt in final homogenization step: $\langle\Delta \varepsilon\rangle_{\omega}=\Delta \bar{\varepsilon}$.

- Initialization in inclusions: $\langle\Delta \varepsilon\rangle_{\omega_{1}}=\langle\Delta \varepsilon\rangle_{\omega}$.

- Loop within each pseudo-grain (iteration index omitted for simplicity):

- Check if solid inclusions or cavities:

(a) if solid inclusions:

Call $J_{2}$ elastoplasticity constitutive box with $\langle\Delta \varepsilon\rangle_{\omega_{1}}$ and $\left.<\varepsilon_{n}\right\rangle_{\omega_{1}}$ as arguments, The box returns $\left.<\sigma_{n+1}\right\rangle_{\omega_{1}}$ and algorithmic tangent $\hat{\mathbf{C}}_{n+1}^{a l g}$;

(b) if cavities, go directly to next step.

- Compute average strain increment in the matrix phase:

$$
<\Delta \varepsilon>_{\omega_{0}}=\frac{<\Delta \varepsilon>-\left(1-v_{0}\right)<\Delta \varepsilon>_{\omega_{1}} .}{v_{0}} .
$$

- Call constitutive box of Ju's damage model with $\langle\Delta \varepsilon\rangle_{\omega_{0}}$ and $<\varepsilon_{n}>_{\omega_{0}}$ as $\hat{\mathrm{C}}_{d}$ arguments. The box returns $\left\langle\sigma_{n+1}\right\rangle_{\omega_{0}}$ and secant operator $\hat{\mathbf{C}}_{n+1}^{d}$.

- Compute tangent operator for Ju's model, $\hat{\mathbf{C}}_{n+1}^{d t g}$.

- Compute the instantaneous Poisson's ratio $v_{t}$ from the isotropic part of $\hat{\mathbf{C}}_{n+1}^{d t g}$.

- Compute Eshelby's tensor $\mathbf{S}\left(v_{t}, A_{r}\right)$.

- Compute strain concentration tensor A using Mori-Tanaka. If solid inclusions:

$$
\mathbf{A}=\left[v_{0}\left\{\mathbf{I}^{s}+\mathbf{S}:\left[\left(\hat{\mathbf{C}}_{n+1}^{d t g}\right)^{-1}: \hat{\mathbf{C}}_{n+1}^{a l g}-\mathbf{I}^{s}\right]\right\}+\left(1-v_{0}\right) \mathbf{I}^{s}\right]^{-1} .
$$

If cavities:

$$
\mathbf{A}=\left[\mathbf{I}^{s}-v_{0} \mathbf{S}\right]^{-1} .
$$

- Check strain compatibility in the inclusions:

$$
<\Delta \varepsilon>_{\omega_{1}}=? \quad \mathbf{A}:<\Delta \varepsilon>_{\omega}
$$

- If yes: no more iterations for this pseudo-grain, 
- Else, perform a new iteration with:

$$
<\Delta \varepsilon>_{\omega_{1}}=\mathbf{A}:<\Delta \varepsilon>_{\omega} .
$$

- After convergence, compute effective tangent operator of the pseudo-grain. If it contains solid inclusions then:

$$
\overline{\mathbf{C}}_{\Omega_{i, p}}^{t g}=\hat{\mathbf{C}}_{n+1}^{d t g}+\left(1-v_{0}\right)\left(\hat{\mathbf{C}}_{n+1}^{a l g}-\hat{\mathbf{C}}_{n+1}^{d t g}\right): \mathbf{A} .
$$

If the pseudo-grain contains cavities, then:

$$
\overline{\mathbf{C}}_{\Omega_{i, p}}^{t g}=\hat{\mathbf{C}}_{n+1}^{d t g}-\left(1-v_{0}\right) \hat{\mathbf{C}}_{n+1}^{d t g}: \mathbf{A} .
$$

Save pseudo-grain's effective tangent and average stresses and strains.

- Compute RVE's effective tangent moduli and macro stresses

$$
\overline{\mathbf{C}}^{t g}=\left\langle\overline{\mathbf{C}}_{\Omega_{i, p}}^{t g}\right\rangle_{i, \psi_{i}}, \quad \Delta \bar{\sigma}=\overline{\mathbf{C}}^{t g}: \Delta \bar{\varepsilon} .
$$

\section{NUMERICAL SIMULATIONS}

In all numerical simulations presented in this section, the steel fibers or particles obey $J_{2}$ elastoplasticity with Young's modulus $E_{I}=210 \mathrm{GPa}$, Poisson's ratio $v_{I}=0.3$, initial yield stress $\sigma_{Y}=210 \mathrm{MPa}$, and power-law isotropic hardening $\left(R(p)=K p^{m}\right)$ with $K=416 \mathrm{MPa}$ and $m=0.3895$.

\section{Validation Against Experimental Results}

From the experimentally obtained load-deflection curves of bent plain concrete beams, a uniaxial tensile stress-strain curve was obtained with the incremental method developed in 'Conversion of the load-deflection curve into a stress-strain curve' section. This curve (plotted in Figure 6) enabled the fitting of the parameters of Ju's brittle damage model ('Micro constitutive models' section). The following values were found: $E=36.9 \mathrm{GPa}, \nu=0.19, k=0.3, A=14.3, B=15.8$, and $\xi_{0}=2.4 \times 10^{-5} \mathrm{MPa}$. The latter four values pertain to the damage evolution function $H(\xi)$, Equation (33). Figure 9 shows that the experimental curve is correctly simulated with the fitted values. The agreement might be improved by considering another expression of $H(\xi)$ in a future work.

Next, the uniaxial macro response of concrete matrix reinforced with 1 and $3 \%$ of randomly oriented short fibers $\left(A_{r}=60\right)$ was numerically simulated using homogenization. Porosity increase resulting from fibers 


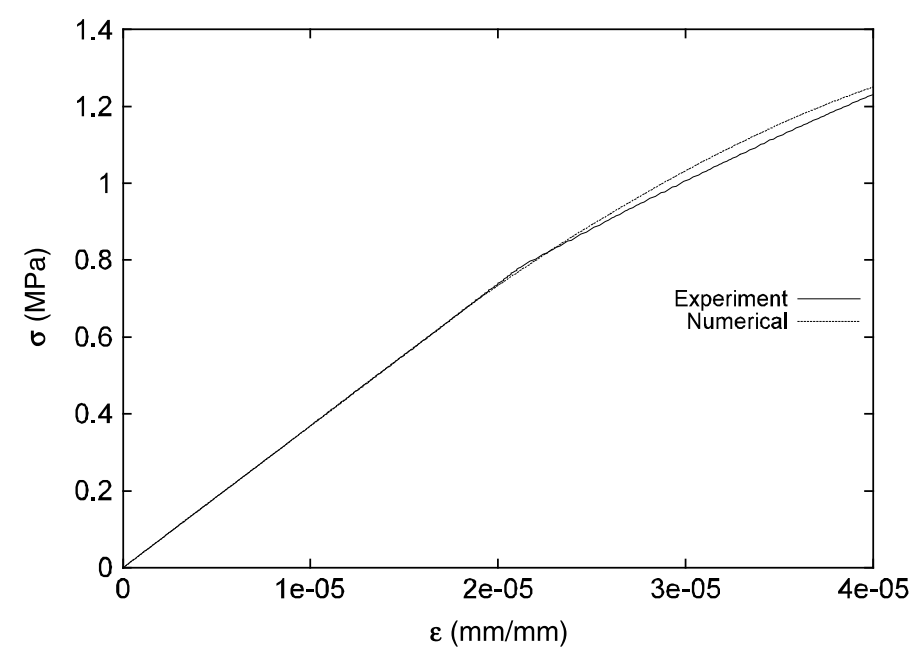

Figure 9. Ju's damage model identification from experimental results.

addition in concrete mix was included as a third phase made of spherical cavities of volume fraction equal to 0.5 and $0.85 \%$, respectively. Those values of porosity were obtained experimentally as follows: what one knows are fibers and plain concrete densities $\rho_{\mathrm{f}}$ and $\rho_{\mathrm{pc}}$, fibers mass $m_{\mathrm{f}}$, and the total volume $V_{\mathrm{T}}$ of SFRC specimen. The volumes $V_{\mathrm{f}}$ of fibers and $V_{\mathrm{pc}}$ of plain concrete materials present in SFRC can then be easily computed with: for fibers $V_{\mathrm{f}}=m_{\mathrm{f}} / \rho_{\mathrm{f}}$ and for plain concrete material $V_{\mathrm{pc}}=m_{\mathrm{pc}} / \rho_{\mathrm{pc}}$ where plain concrete mass $m_{\mathrm{pc}}$ is evaluated with: $m_{\mathrm{pc}}=m_{\mathrm{T}}-m_{\mathrm{f}}$. The volume of cavities is then deduced with: $V_{\text {cav }}=V_{\mathrm{T}}-V_{\mathrm{pc}}-V_{\mathrm{f}}$. The numerical predictions are validated against the experimental results in Figures 10 and 11, and a good agreement can be observed.

In the version of Ju's damage model implemented in this article, the nonlinearity in the tensile stress-strain response of plain concrete is only due to the damage evolution. Therefore, for a uniaxial stress state, a damage measure $d$ can be defined simply by the decrease in secant stiffness, similarly to the well-known Lemaitre and Chaboche (1990) model:

$$
d=1-\frac{E_{d}}{E}, \quad d \in[0,1]
$$

The same definition can be applied to the composite material under uniaxial macro stress. The evolution of $d$ as a function of tensile strain is plotted in Figure 12 for plain and reinforced concrete. 


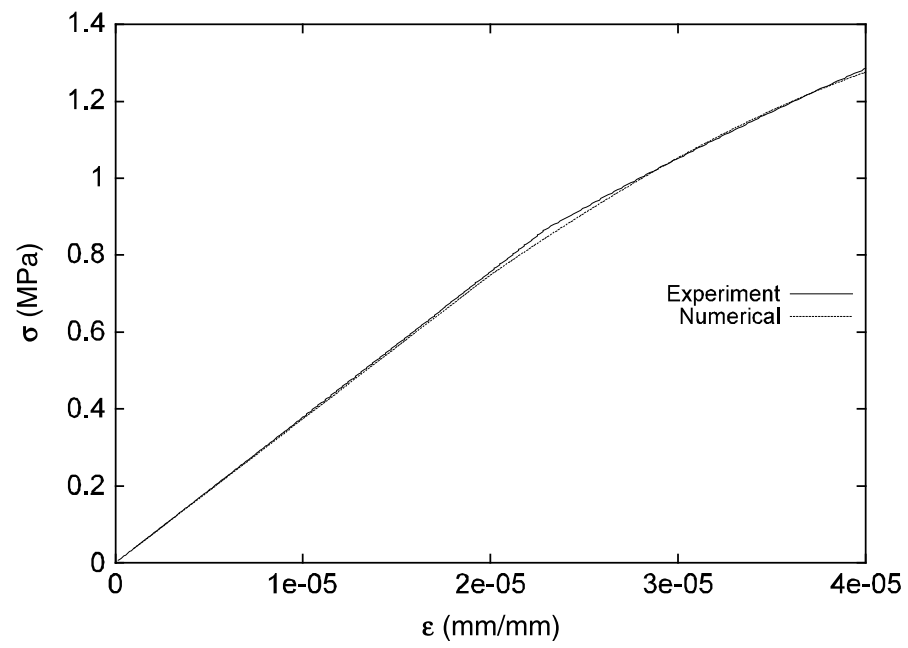

Figure 10. SFRC with $1 \%$ of fibers under uniaxial tension: experiment vs numerical results.

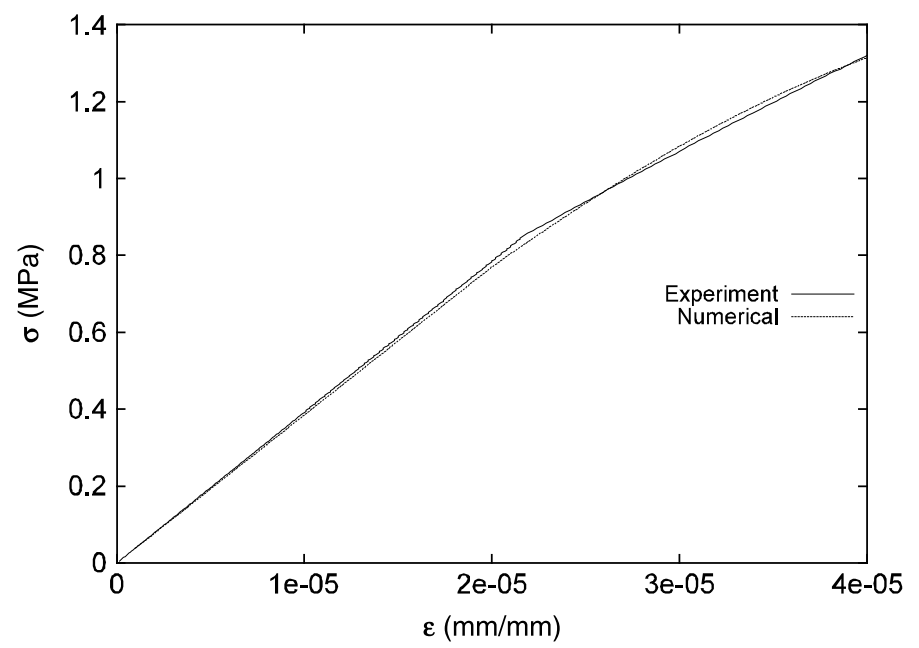

Figure 11. SFRC with $3 \%$ of fibers under uniaxial tension: experiment vs numerical results.

\section{Unreinforced Concrete-like Material}

The authors consider a fictitious plain concrete-like brittle material whose parameters are: $E=25 \mathrm{GPa}, \nu=0.2, k=0.7, A=14.3, B=14.8$, and $\xi_{0}=2.4 \times 10^{-5} \mathrm{MPa}$. 


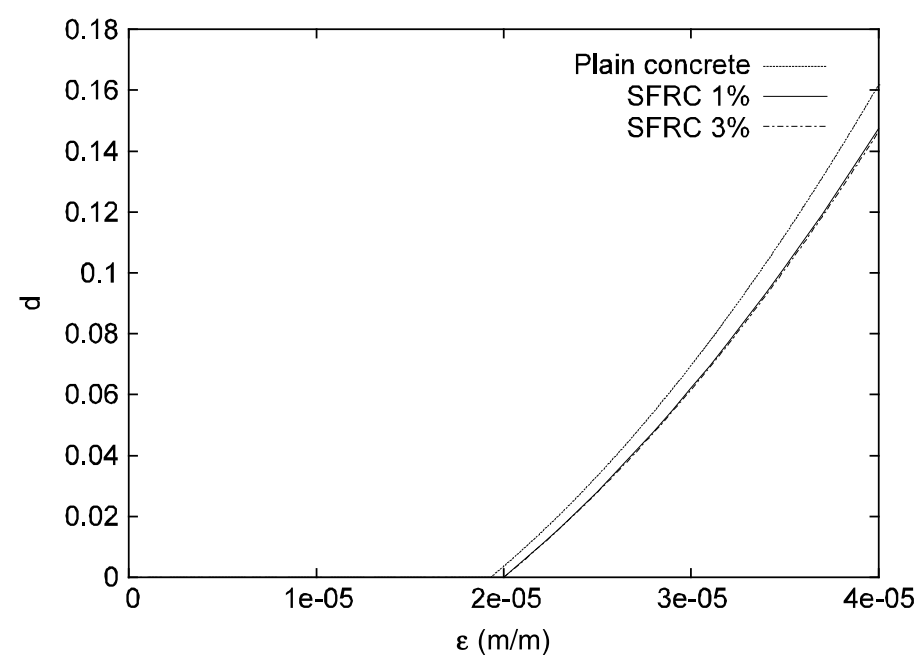

Figure 12. Damage evolution of plain concrete and SFRC under uniaxial tensile loading.

The first example simulates a uniaxial tension test with three different strain increments: $\Delta \bar{\varepsilon}=\bar{\varepsilon} / 10, \bar{\varepsilon} / 100$, and $\bar{\varepsilon} / 1000$. The results are plotted in Figure 13 and do not show a significant influence of the strain increment values on the converged response.

The second example considers the same material data except $\xi_{0}=2.4 \times 10^{-2} \mathrm{MPa}$ and studies the uniaxial response under cyclic strain history. After a first cycle, the peak strains are increased (in absolute values) in the second cycle. The stress-strain response and the strain history are displayed in Figures 14 and 15(a), respectively. Figure 14 shows that under tensile loading, the material first displays an elastic behavior then undergoes damage. Both in unloading and compressive stages, the material's response is linear with a slope corresponding to the elastic damaged modulus. The material remains in the elastic domain until the previously reached damage threshold is attained again in tension. Those features are also reproduced by the scalar damage variable defined in Equation (36) whose time-evolution is depicted in Figure 15(b). It is also interesting to point out that the initial stiffness of the material is not recovered in the compression stage. This does not totally correspond to the truly observed behavior since during the unloading stage following the tension one, microcracks closure leads to a partial recovery of the initial stiffness. Examination of damage operator evolution expression (Equation (35)) and its time-discretization (equation in step 9 of the algorithm proposed in 'Ju's unilateral damage model' section) of Ju's model that the authors have implemented, clearly indicates that the recovery of the initial stiffness is not accounted for. In other words, 


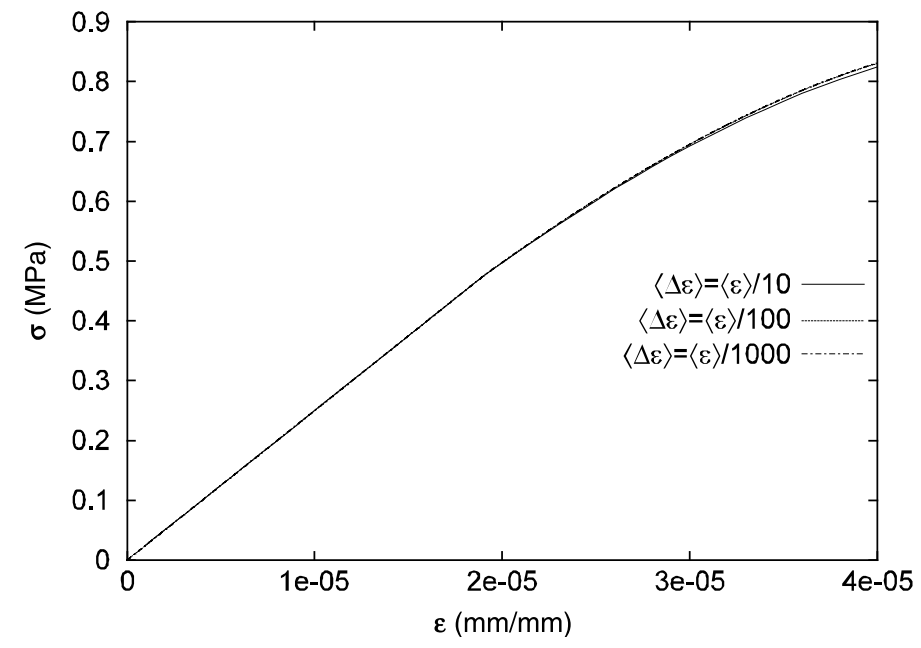

Figure 13. Convergence of Ju's model under various strain steps.

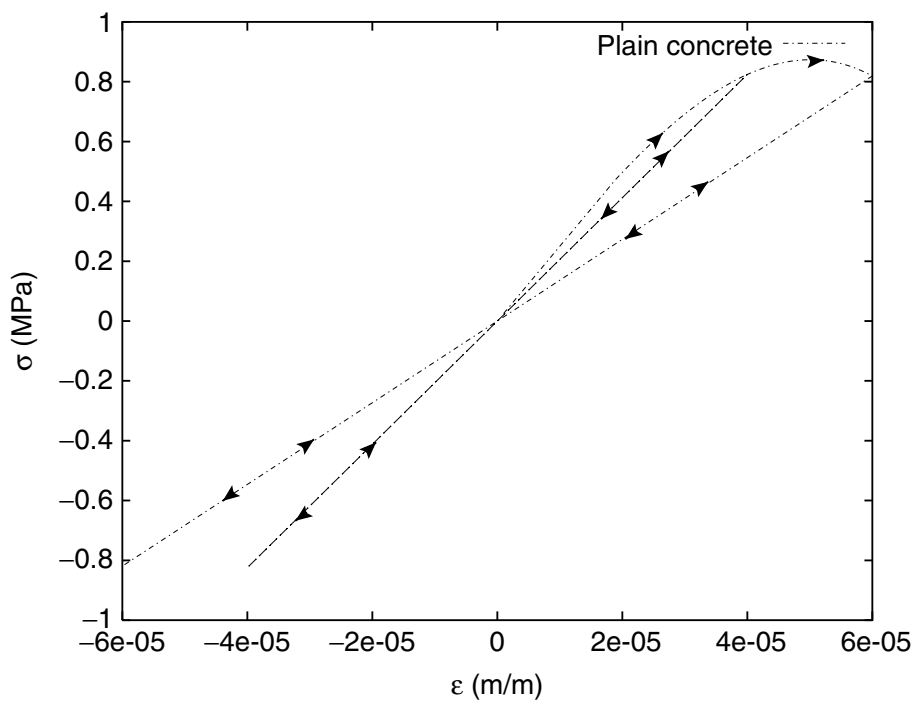

Figure 14. Concrete-like matrix under cyclic loading (traction/compression): strain-stress curves.

even if damage does not evolve there is no deactivation of damage effects when the specimen undergoes compressive loading. Note that this has the advantage of avoiding the possible discontinuities of the stress-strain response. 

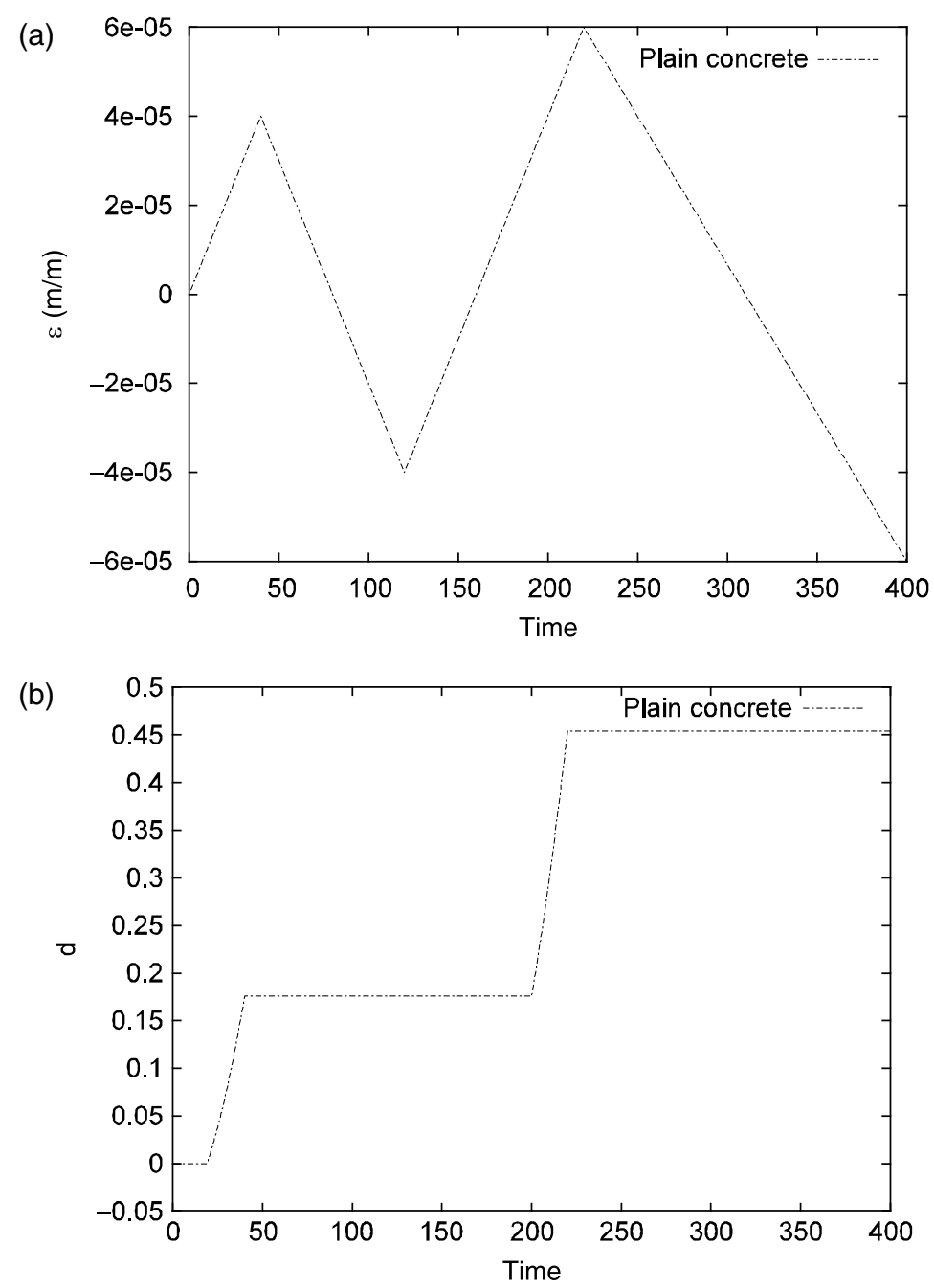

Figure 15. Damage evolution of concrete-like matrix under uniaxial cyclic loading (traction/ compression).

\section{Particle- and Fiber-reinforced Concrete-like Matrix Materials}

The authors consider a fictitious concrete-like material which is reinforced with either steel particles or fibers. These reinforcements lead to composite materials designated as steel particle-reinforced concrete (SPRC) and SFRC, respectively. The unreinforced matrix material has the same properties as in the previous section except when indicated otherwise. 


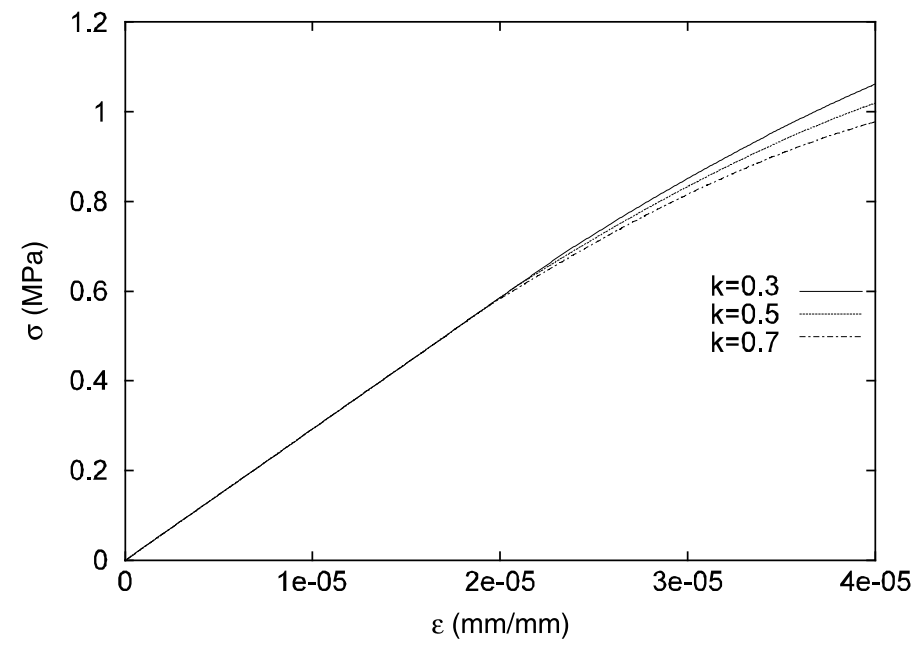

Figure 16. Influence of crack density factor ' $k$ ' on the macro-response of SPRC under tensile loading.

In the first example, the authors study the influence of the matrix' microcrack density parameter $k$ - Equation (33) - which takes 3 values: $k=0.3,0.5$, and 0.7 . Spherical particles of volume fraction $v_{1}=10 \%$ are embedded in the matrix. The influence of $k$ on the composite's response is displayed in Figure 16 which shows that the composite softens with increasing values of $k$.

In the second example, the authors illustrate the influence of the reinforcements' shape. The matrix' parameter $k$ is set to $k=0.7$ and elastic steel inclusions $\left(v_{1}=10 \%\right)$ are considered in three shapes: spherical $\left(A_{r}=1\right)$, long $\left(A_{r}>100\right)$, and short $\left(A_{r}=50\right)$ fibers. The fibers are aligned in the macro tension direction. As expected, Figure 17 shows that the composite stiffens with increasing values of the aspect ratio $A_{r}$.

In the third example, the authors investigate the influence of the matrix' parameter $A$ - Equation (33) - which takes three values: $A=10,14.3$, and 17. The matrix is reinforced with spherical steel particles $\left(v_{1}=10 \%\right)$. Simulations of uniaxial tension tests show that the composite softens considerably with increasing values of $A$ (Figure 18).

The same composite is considered in the fourth example which illustrates the influence of the matrix' parameter $B$ which now takes three values $B=10,14.8$, and 17. Figure 19 shows that the composite softens dramatically with increasing values of $B$.

In the fifth example, the response of SFRC under cyclic loading is simulated. The parameters of the matrix material are: $E_{m}=20 \mathrm{GPa}$, 


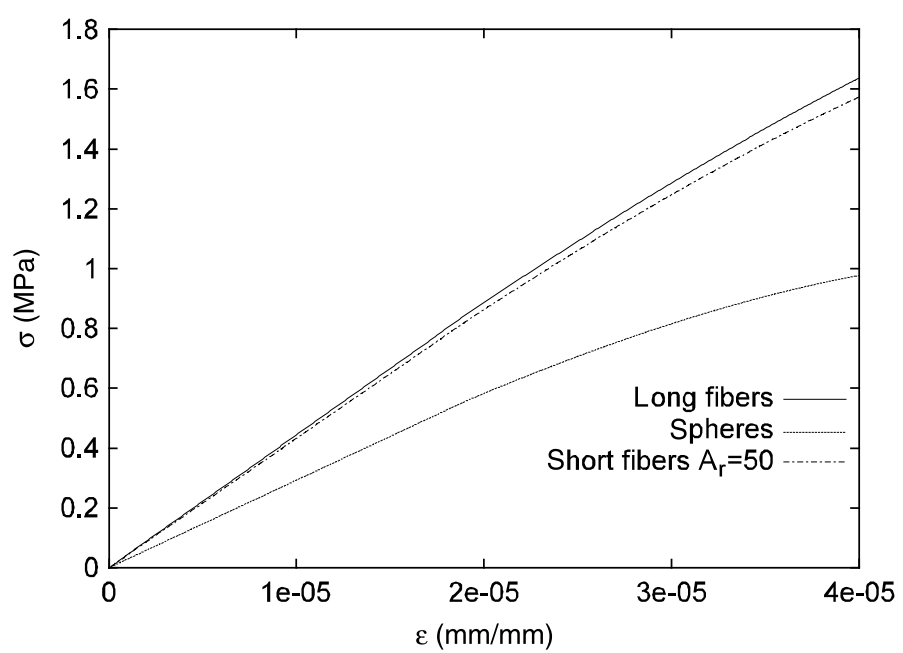

Figure 17. Influence of the shape of the reinforcement on the response of reinforced concrete matrix submitted to tension.

$v_{m}=0.2, A=0.8, B=0.16, k=0.7$, and $\xi_{0}=2.4 \times 10^{-2} \mathrm{MPa}$. The matrix is reinforced with short steel fibers $\left(A_{r}=50\right.$ and $\left.v_{1}=20 \%\right)$ which are elastoplastic with linear isotropic hardening $(m=1)$. The composite is subjected to cyclic tension/compression in the fiber direction under imposed macro strain. The resulting stress-strain response is plotted in Figure 20 together with those of the fibers and matrix taken separately. Both elastoplastic and damage phenomena are illustrated. First, the composite behaves elastically (step 1), then when the yield stress hardening of the fibers is reached, the response of the composite becomes elastoplastic, step 2. Under higher tensile strain, the composite undergoes damage, step 3. During unloading and compressive loading phases, damage does not evolve, the composite exhibits elastic and then elastoplastic behavior: steps 4 and 5, respectively. During step 6, the composite goes elastic, and then elastoplastic under tension (step 7). No more damage develops in this step since the damage threshold is not reached.

\section{Validation Against Unit Cell FE Results}

The mechanical response of a sphere-reinforced composite with a periodic microstructure is studied. Figure 21 illustrates the different steps of the transition from a $3 \mathrm{D}$ representation to a $2 \mathrm{D}$ axisymmetric unit cell model. Zero displacements are imposed in the radial direction to the left vertical 


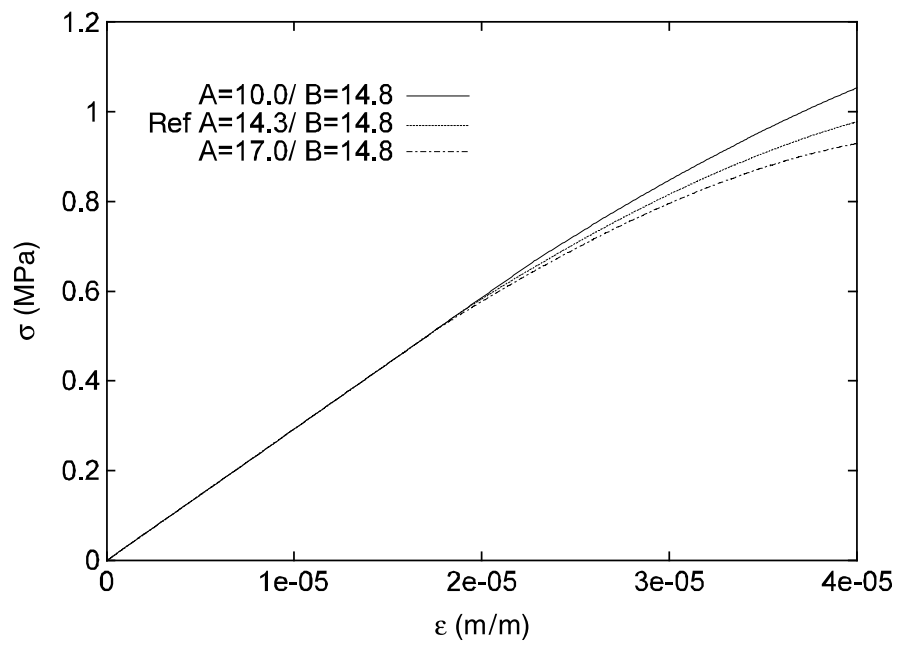

Figure 18. Influence of material parameter $A$ on the response of $10 \%$ steel spherereinforced concrete submitted to tension.

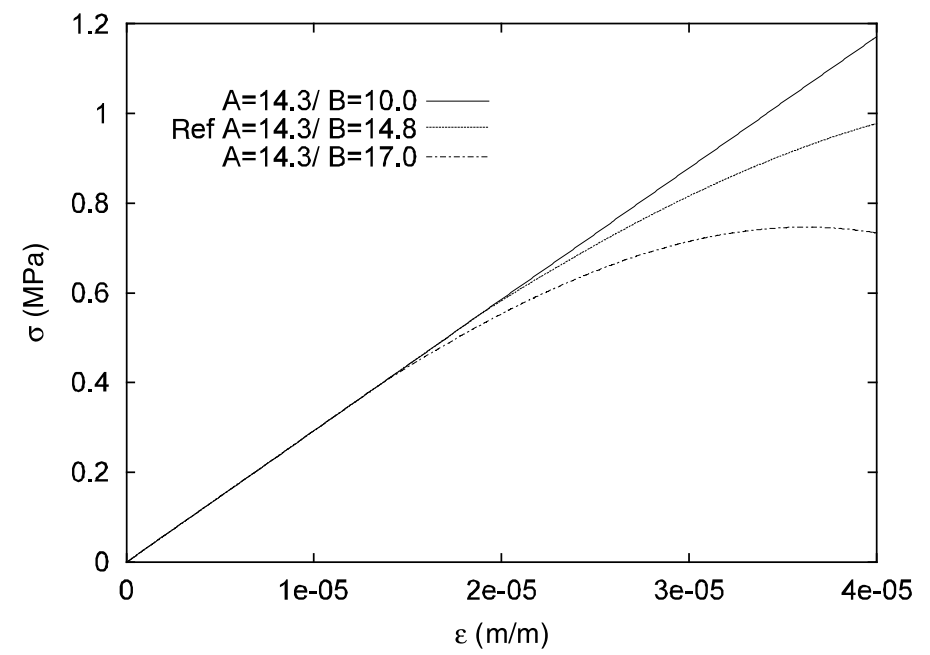

Figure 19. Influence of material parameter $B$ on the response of $10 \%$ steel spherereinforced concrete submitted to tension.

side and in the vertical direction to the bottom horizontal side. The right vertical side is constrained to have uniform radial displacement. Uniform vertical displacement is imposed on the top horizontal side. The unreinforced matrix material obeys Ju's brittle damage model with the 


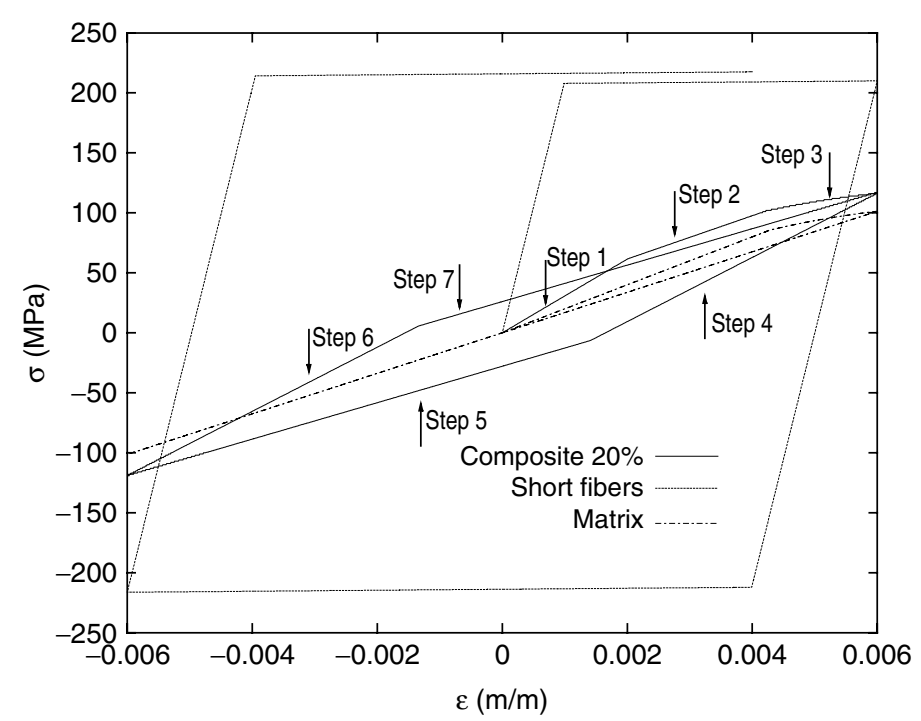

Figure 20. SFRC under cyclic loading: effect of damage and plasticity on the macroscopic response of the composite.

experimentally identified parameters of the 'Validation against experimental results' section. The model was integrated into the ABAQUS FE program via a user-defined UMAT subroutine. The matrix is reinforced with $v_{1}=10 \%$ of elastic steel particles of properties $E_{I}=210 \mathrm{GPa}$ and $v_{\mathrm{I}}=0.3$. The unit cell was meshed with 646 axisymmetric CAX3R elements and 357 nodes.

A uniaxial macro tension test is simulated with two completely different methods: (i) a unit cell FE analysis and (ii) Mori-Tanaka homogenization. Figure 22 shows a good agreement between the macro stress-strain curves predicted by the two methods.

\section{Validation of a Two-scale Approach Against the Experimental Bending Test}

The authors developed a two-scale approach which enables the numerical simulation within reasonable CPU time, memory usage, and user time of realistic structures made of composite materials and subjected to complex loadings. For that purpose, the authors homogenization code was integrated into ABAQUS throughout its UMAT interface. At each iteration of the global equilibrium equations of the FE analysis and at each integration point of the macro FE mesh, the homogenization module is called. 


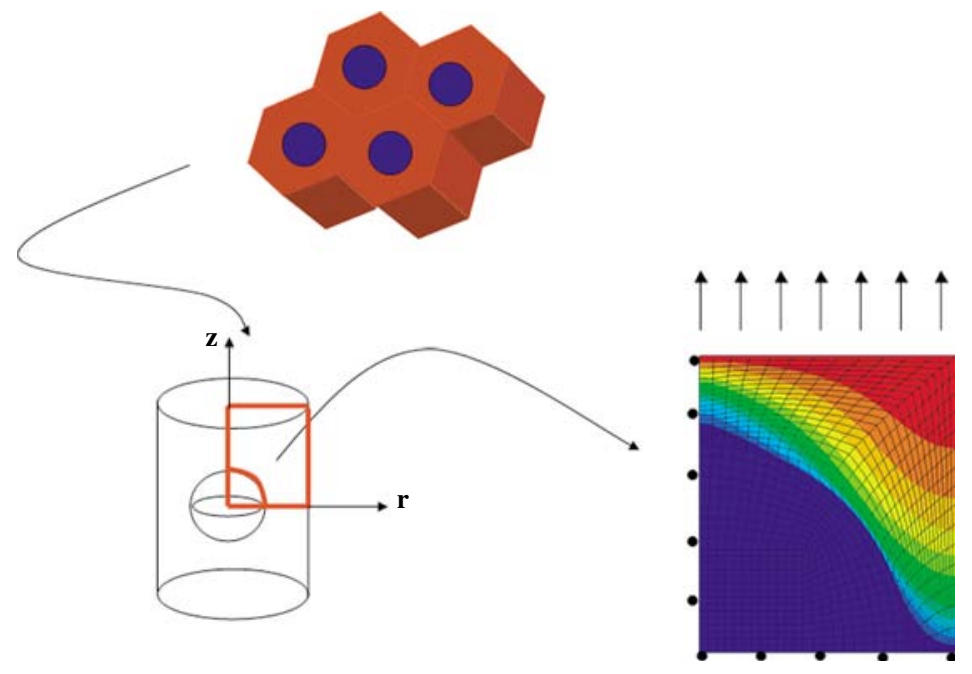

Figure 21. A $2 D$ unit cell representation of a composite with periodic microstructure.

ABAQUS passes the macrostrain at time $t_{n}$, the macrostrain increment, material parameters and history variables at time $t_{n}$. The homogenization module computes the macro-stress $\bar{\sigma}$ and the homogenized macrotangent modulus $\overline{\mathbf{C}}^{\text {tg }}$ and returns them to ABAQUS.

The aim of the following example is to numerically simulate the fourpoint bending test which was performed experimentally (the 'Experimental bending tests' section). The SFRC beam contains 3\% of randomly oriented steel fibers and $0.85 \%$ of spherical cavities. The concrete matrix follows Ju's damage model with the experimentally identified parameter values of the 'Validation against experimental results' section. The beam was meshed with 400 brick elements of type C3D8R and 612 nodes (Figure 23). The displacements (not the rotations) of the nodes located on the right support are zero while only the vertical displacement of the node located on the left support is imposed to be zero. At the upper face of the beam a vertical displacement $(0.02 \mathrm{~mm})$ is applied throughout a rigid body. The numerically obtained load-deflection curve is plotted in Figure 24 and superposed to the experimental curve. A fair agreement is observed although the linear elastic slopes are somewhat different for the two curves and damage occurs much later in the numerical simulation. A better agreement might be obtained with a better identification of Ju's model based on a damage evolution function different from the one considered here - Equation (33). It should be interesting to note that in some cases, simulations obtained using the homogenization code could give results that are too stiff, compared to those 


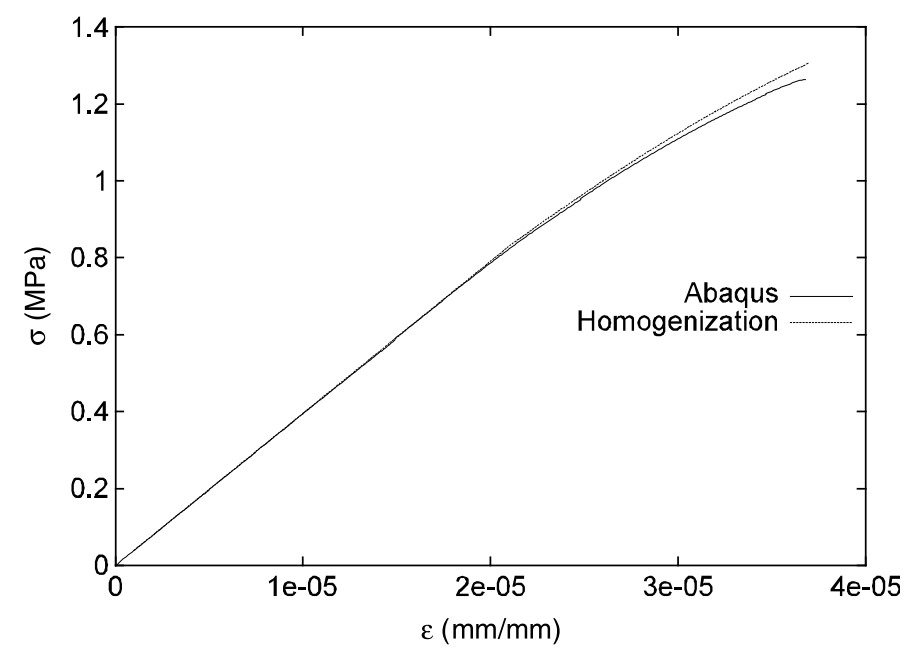

Figure 22. Steel sphere-reinforced concrete under tension: unit cell FE computation vs homogenization results.

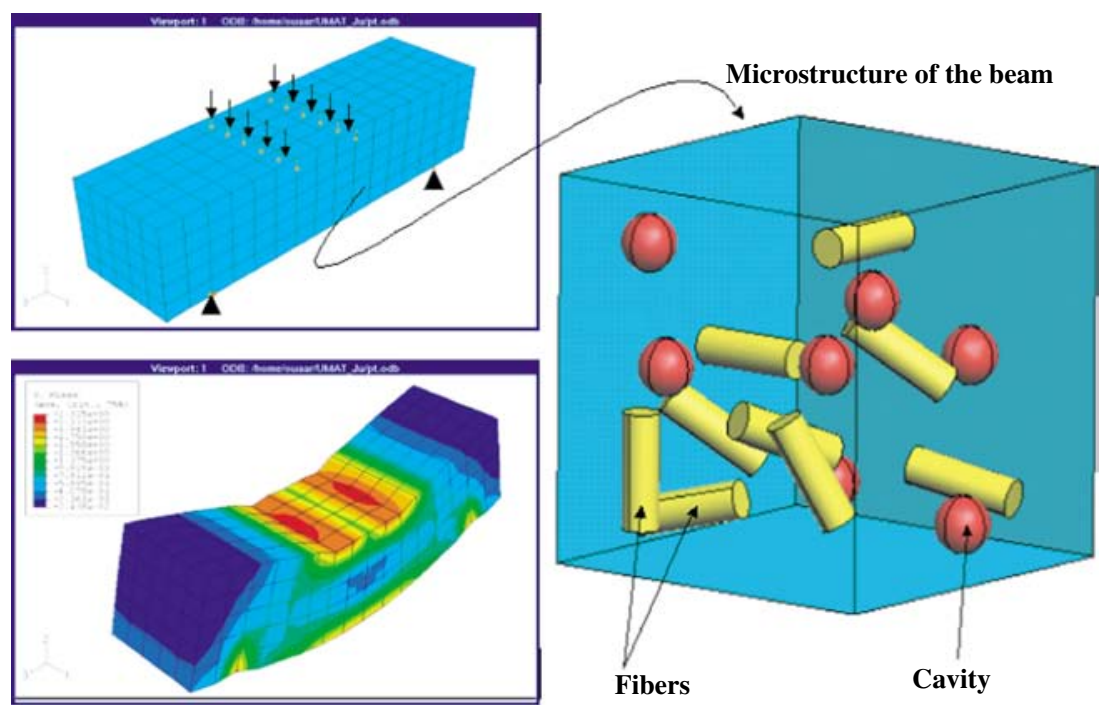

Figure 23. $3 D$ modeling of a beam submitted to a four-point bending test: a two-scale numerical approach.

obtained by FE computations (Figures 22 and 24). This is due to the fact that the homogenization model is derived within the framework of the mean-field approach which does not account for strain fluctuations in each phase. The sole information used to compute the reference tangent moduli 


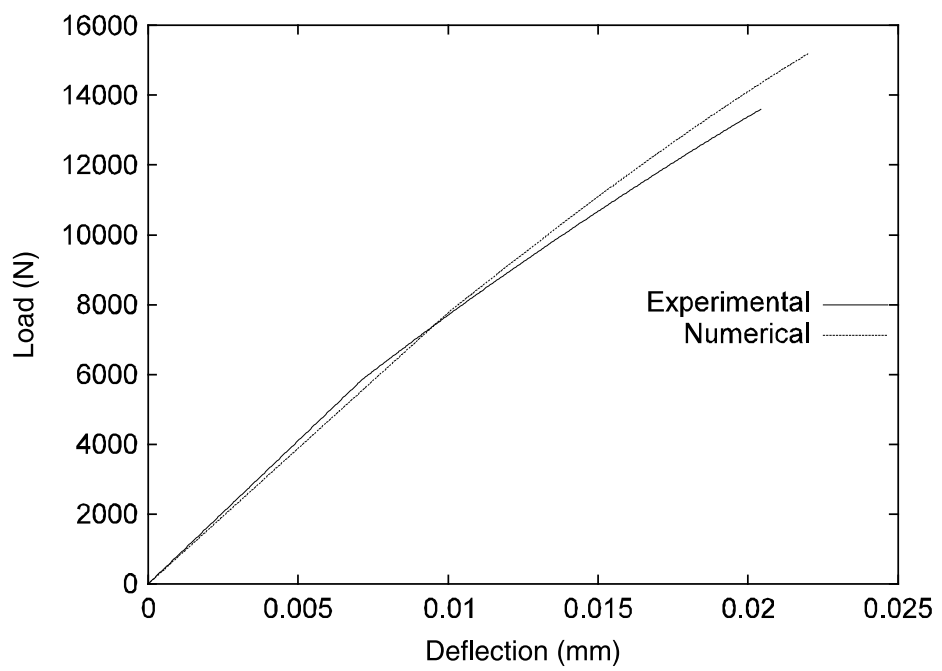

Figure 24. SFRC (3\% of fibers) under bending: load-deflection curve, numerical vs experimental results.

per phase is the average strain of the considered phase. The results predicted by the authors' model can be improved by the authors' considering the second-moment of strain for instance.

\section{CONCLUSIONS}

Experiments, micromechanical modeling, and numerical simulations of SFRC were presented in this article. In the experimental part, four-point bending tests were conducted on prismatic specimens made of plain concrete and SFRC with different volume fractions of fibers. To be more useful, the load-deflection curves were converted to stress-strain curves with the help of a method based on an incremental formulation which the authors have developed in the nonlinear regime. In a second part of this article, the authors extended mean-field homogenization schemes to nonlinear inelasticity following a Hill-type step-by-step formulation which enables the simulation of various loading histories including unloading and nonradial paths. Two rate-independent constitutive models were implemented at the microlevel: Ju's brittle damage model for the concrete matrix and $J_{2}$ elastoplasticity for steel fibers. Eshelby's tensor was computed by using an isotropic part of the nonlinear tangent operator of the reference matrix material. To take into account the porosity, SFRC was considered as a three-phase composite and modeled using a two-step Mori-Tanaka/Voigt homogenization procedure. 
Several discriminating tests on both plain concrete and SFRC composites were simulated: monotonic tension, unloading, and cyclic loadings. The accuracy of the proposed approach was validated against both direct FE computations and experimental results. For FE simulations the authors considered a periodic microstructure represented by an axisymmetric unit cell. The homogenization code was also interfaced to the FE package ABAQUS with the help of a user-defined material interface in order to perform, the two-scale simulations of 3D realistic structures. In many cases, the authors' predictions show a good agreement with both FE simulations and experimental results.

From the experimental point of view, it was found that adding fibers to concrete matrix does not increase the stiffness of the composite in a significant manner. However, the fibers improve the ductility, and when analyzing the load-displacement curve of the SFRC specimen, one can see a considerable increase of the area located between the curve and the deflection axis. In addition, the authors have also observed that when fibers are added to a concrete mix, porosity increases. This observation strongly suggests limitation of the volume fraction of fibers in SFRC. It seems that the apparition of porosity is responsible for the limited stiffness improvement of SFRC. The porous phase must be seen as a component of the microstructure of the composite, not as a consequence of loadinduced damage.

As future work, it will be interesting to develop a micromechanical approach for macrocrack propagation which will facilitate study of the ductile behavior of SFRC and contrast it with the brittle behavior of unreinforced concrete. Fiber-matrix debonding is also an important aspect to be investigated and taken into account in a micromechanical modeling.

\section{ACKNOWLEDGMENTS}

The authors gratefully acknoweldge the support of the Belgian Federal Science Policy, IUAP P5/08 project 'From microstructure towards plastic behaviour of single- and multi-phase materials,' and Région Wallonne through the WINNOMAT project (contract 415660).

\section{REFERENCES}

ABAQUS (2004). A General Purpose Finite Element Program, Abaqus, Inc., Pawtucket, RI, USA.

Benveniste, Y. (1987). A New Approach to the Application of Mori-Tanaka's Theory in Composite Materials, Mechanics of Materials, 6: 147-157. 
Bornert, M., Bretheau, T. and Gilormini, P. (eds), (2001). Homogénéisation en mécanique des matériaux, 1. Matériaux aléatoires élastiques et milieux périodiques, HERMES Science, Paris.

Camacho, C.W., Tucker, C.L. III, Yalvac, S. and McGee, R.L. (1990). Stiffness and Thermal Expansion Predictions for Hybrid Short Fiber Composites, Polymer Composites, 11(4): 229-239.

Chaboche, J.-L. (1992). Damage Induced Anisotropy: On the Difficulties Associated with the Active/Passive Unilateral Condition, Internat. Journal of Damage Mechanics, 1: $148-171$.

Doghri, I. (2000). Mechanics of Deformable Solids: Linear, Nonlinear, Analytical and Computational Aspects, Springer-Verlag, Berlin.

Doghri, I. and Friebel, C. (2005). Effective Elasto-plastic Properties of Inclusion-reinforced Composites. Study of Shape, Orientation and Cyclic Response, Mechanics of Materials, 37: $45-68$.

Doghri, I. and Ouaar, A. (2003). Homogenization of Two-phase Elasto-plastic Composite Materials and Structures. Study of Tangent Operators, Cyclic Plasticity and Numerical Algorithms, Internat. Journal of Solids and Structures, 40: 1681-1712.

Doghri, I. and Tinel, L. (2005). Micromechanical Modeling and Computation of Elasto-plastic Materials Reinforced with Distributed Orientation Fibers, Internat. Journal of Plasticity, 21(10): 1919-1940.

Eshelby, J.D. (1957). The Determination of the Elastic Field of an Ellipsoidal Inclusion, and Related Problems, Proc. Roy. Soc. London-Ser. A, 241: 376-396.

Gavazzi, A.C. and Lagoudas, D.C. (1990). On the Numerical Evaluation of Eshelby's Tensor and its Application to Elastoplastic Fibrous Composites, Computational Mechanics, 7: 13-19.

Hill, R. (1965). A Self Consistent Mechanics of Composite Materials, J. Mech. Phys. Solids, 13: $213-222$.

Ju, J.W. (1989). On Energy-based Coupled Elastoplastic Damage Theories: Constitutive Modeling and Computational Aspects, Int, J. Solids Structures, 25(7): 803-833.

Krajcinovic, D. (1996). Damage Mechanics, North-Holland series in Applied Mathematics and Mechanics. Amsterdam.

Lemaitre, J. and Chaboche, J.-L. (1990). Mechanics of Solid Materials, Cambridge University Press, Cambridge, England, translation of the French edition, Dunod and Bordas, Paris, 1985.

Li, G., Zaho, Y. and Pang, S.-S. (1999). Four-phase Sphere Modeling of Effective Bulk Modulus of Concrete, Cement and Concrete Research, 29: 839-845.

Lielens, G. (1999). Micro-macro Modeling of Structured Materials, PhD Dissertation, UCL/FSA, Louvain-la-Neuve, Belgium.

Mori, T. and Tanaka, K. (1973). Average Stress in Matrix and Average Elastic Energy of Materials with Misfitting Inclusions, Acta Materiala, 21: 571-574.

Ouaar, A., Doghri, I. and Thimus, J.Fr. (2000). Modeling and Simulation of Fiber-reinforced Concrete using Homogenization Schemes, 5th National Congress on Theoretical and Applied Mechanics, 355-358, L-L-N, Belgium.

Ouaar, A., Doghri, I. and Thimus, J.Fr. (2003). Micro-mechanics of Two-phase Nonlinear Composite Materials, Application to Reinforced Mortar and Concrete: Modeling and Simulation, 6th NCTAM, 2003-48, Ghent.

Pierard, O., Friebel, C. and Doghri, I. (2004). Mean-field Homogenization of Multi-phase Thermo-elastic Composites: A General Framework and its Validation, Composites Science and Technology, 64/10-11: 1587-1603.

Rossi, P. (1998). Les bétons de fibres métalliques, Presses de l'ENPC, Paris. 
Soroushian, P. and Bayasi, Z. (1991). Fiber-type Effects on the Performance of Steel Fiberreinforced Concrete, ACI Materials Journal, title no. 88-M16, pp. 129-134, March-April 1991.

Teng, T.L., Chu, Y.A., Chang, F.A. and Chin, H.S. (2004). Calculating the Elastic Moduli of Steel-fiber Reinforced Concrete using a Dedicated Empirical Formula, Computational Materials Science, 31: 337-346.

Van Hauwaert, A. (2000). Fiber-matrix Adhesion in Steel Fiber Reinforced Concrete, PhD Dissertation, UCL/FSA, Louvain-la-Neuve, Belgium, March 2000.

Walkus, B.R., Januszkiewicz, A. and Jeruzal, J. (1979). Concrete Composite with Cut Steel Fiber Reinforcement Subjected to Uniaxial Tension, ACI Materials Journal, Title No. 76-45: 1079-1092, October 1979.

Zaho, X.-H. and Chen, W.F. (1998). The Effective Elastic Moduli of Concrete and Composite Materials, Composites Part B, 29B: 31-40. 